Article

\title{
Rhodium(III)-Catalyzed Redox-Neutral [3+3] Annulation of $N$-nitrosoanilines with Cyclopropenones: A Traceless Approach to Quinolin-4(1H)-One Scaffolds
}

\author{
Lingjun Liu ${ }^{1,2}$, Jiyuan $\mathrm{Li}^{2}$, Wenhao Dai ${ }^{1,2}$, Feng Gao ${ }^{2}$, Kaixian Chen ${ }^{1,2,3, *}$, Yu Zhou ${ }^{2, *}$ and \\ Hong Liu 1,2,3,*
}

1 State Key Laboratory of Natural Medicines and Department of Medicinal Chemistry, China Pharmaceutical University, 24 Tong Jia Xiang, Nanjing 210009, China; engrossment_llj@163.com (L.L.); dwh1992@163.com (W.D.)

2 State Key Laboratory of Drug Research and Key Laboratory of Receptor Research, Shanghai Institute of Materia Medica, Chinese Academy of Sciences, 555 Zu Chong Zhi Road, Shanghai 201203, China; lijiyuan@shu.edu.cn (J.L.); zjsygao@163.com (F.G.)

3 Open Studio for Druggability Research of Marine Natural Products, Pilot National Laboratory for Marine Science and Technology (Qingdao), 1 Wenhai Road, Aoshanwei, Jimo, Qingdao 266237, China

* Correspondence: kxchen@simm.ac.cn (K.C.); zhouyu@simm.ac.cn (Y.Z.); hliu@simm.ac.cn (H.L.); Tel.: +86-21-5080-7042 (H.L.)

Received: 29 November 2019; Accepted: 3 January 2020; Published: 9 January 2020 updates

\begin{abstract}
A traceless approach to quinolin- $4(1 H)$-one scaffolds through $\mathrm{Rh}(\mathrm{III})$-catalyzed redox-neutral [3+3] cyclization of $N$-nitrosoanilines with cyclopropenones has been achieved. This protocol features short reaction time and atom-economical combination without extra additives, which can be further applied in the construction of privileged heterocyclic compounds in pharmaceutical chemistry.
\end{abstract}

Keywords: Rhodium(III); redox-neutral; [3+3] annulation; $N$-nitrosoaniline; cyclopropenones; quinolin-4(1H)-ones

\section{Introduction}

Quinolin-4(1H)-ones are ubiquitously present in numerous natural products and drugs, representing an important class of privileged structures in medicinal chemistry [1-5], such as antibiotics norfloxacin and gatifloxacin, a HIV integrase inhibitor, elvitegravir, and a modulator of ATP-binding cassette transporters, lvacaftor (Figure 1). Therefore, the development of highly efficient protocols both in transition-metal-catalyzed C-H activation and photocatalytic methods for the construction of such $N$-heterocyclic scaffolds is an extremely hot issue in modern organic chemistry [6-12]. However, the existing methodologies usually require elaborate-to-access starting materials, multiple steps, or harsh reaction conditions, failing to implement a wide range of applications. Given the importance of quinolin-4(1H)-ones with broad biological activities, there still remains the need to develop efficient, step- and atom-economic synthetic strategies. 
<smiles>CCN1CC(C(=O)O)C(=O)c2cc(F)c(N3CCNCC3)cc21</smiles>

Norfloxacin<smiles>COc1c(N2CCNC(C)C2)c(F)cc2c1N(C1CC1)CC(C(=O)O)C2=O</smiles>

Gatifloxacin<smiles>COc1cc2c(cc1Cc1cccc(Cl)c1F)C(=O)C(C(=O)O)CN2C(CO)C(C)C</smiles>

Elvitegravir<smiles>CC(C)(C)c1ccc(NC(=O)C2CNc3ccccc3C2=O)c(C(C)(C)C)c1O</smiles>

Ivacaftor

Figure 1. Representative drugs containing quinolin-4(1H)-ones.

In the past decade, transition-metal-catalyzed redox-neutral C-H activation reactions have emerged as a robust and versatile methodology, avoiding stoichiometric amounts of external oxidants [13-15]. Recently, $N$-nitroso [16-18] as a novel directing group has aroused increasing attention and has been successfully employed in transition-metal (e.g., Pd, Rh, etc.) catalyzed C-H functionalization (Scheme 1a) [19-22]. In 2013, Zhu's group reported the pioneering work of $\mathrm{Rh}(\mathrm{III})$-catalyzed redox-neutral [3+2] annulation of $\mathrm{N}$-nitrosoanilines with internal alkynes to form efficiently indole derivatives (Scheme 1b) [23-28]. Similarly, several formal [3+2] annulations between $N$-nitrosoanilines and diazo compounds [29] as well as propargyl alcohols [30] utilizing the $N$-nitroso group as an internal oxidant have been reported to prepare diversified indole scaffolds, in which the substrate involving the $\mathrm{N}$-nitroso group seems to be an excellent synthon to build these intriguing privileged structures via a $\mathrm{C}-\mathrm{H}$ bond activation and further annulation cascade. Therefore, in continuation of our recent efforts on transition-metal-catalyzed C-H annulations for the construction of heterocyclic scaffolds [31-36], we surprisingly found a new redox-neutral [3+3] annulation of $N$-nitrosoanilines with cyclopropenones [37-39] to generate a different substituted quinolin-4(1H)-one scaffold (Scheme 1c), which is a desirable privileged structure for further drug discovery. However, coincidentally, a similar work was reported by Cheng [40] after our work was finished and ready to submit. Compared with Cheng's strategy, this method without extra additives also enables the efficient preparation of quinolin- $4(1 \mathrm{H})$-ones in a much shorter time $(2 \mathrm{~h}$ vs. $12 \mathrm{~h})$, and has a good substrate scope.

(a) Transition-metal-catalyzed ortho $\mathrm{C}$-H functionalization of $\mathbf{N}$-nitrosoaniline derivatives

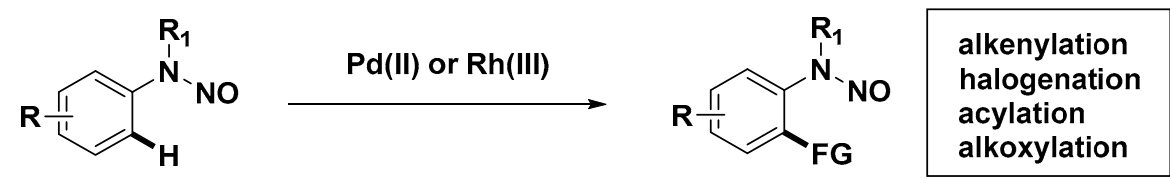

(b) Transition-metal-catalyzed formal [3+2] annulation of $\boldsymbol{N}$-nitrosoaniline derivatives

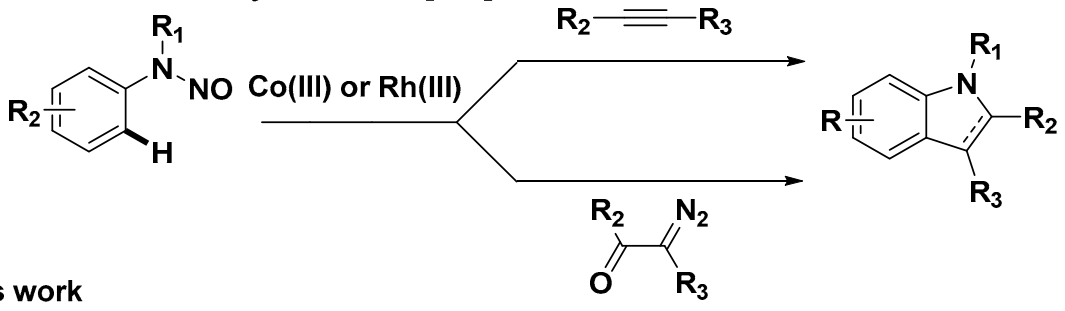

(c) This work<smiles>[R]N([O-])c1cc[R1]cc1</smiles><smiles>[R]c1c([R])c1=O</smiles>

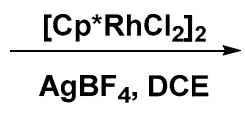<smiles></smiles>

Scheme 1. Transition-metal-catalyzed C-H functionalization of $N$-nitrosoanilines. 


\section{Results and Discussions}

We initiated our studies by examining the reaction conditions of the coupling of $N$-nitrosoaniline, 1a, with diphenylcyclopropenone, 2a, in the presence of a Rh(III) catalyst. As shown in Table 1 , three $\mathrm{Rh}(\mathrm{III})$ catalysts were firstly explored in dichloroethane (DCE), and the desired product, 3a, could only be afforded in $13 \%$ yield under the presence of $\left[\mathrm{Cp}^{*} \mathrm{RhCl}_{2}\right]_{2}$, whereas the other two $\mathrm{Rh}$ (III) catalysts or Rh(III)-free were not effective (Table 1, entries 1-4). The structure of 3a was also unambiguously confirmed by an X-ray crystallographic analysis (see the Supplementary Material for details). However, further explorations demonstrated that a large amount of side product of dimerization of cyclopropenone $[41,42]$ was generated simultaneously in this transformation, which resulted in a low yield of the desired product. Based on these results, we wondered whether lowering the concentration of cyclopropenone could inhibit the formation of the dimerization side product. To our delight, when the concentration was reduced from $0.1 \mathrm{M}$ to $0.02 \mathrm{M}$, the yield of the desired product was increased dramatically, increasing the yield of $3 \mathbf{a}$ to $72 \%$ (entries 5,6 ). Inspired by the results, we further screened the silver salts and the results revealed that $\mathrm{AgBF}_{4}$ was still the most effective, while no desired product was formed in the absence of the silver additive (entries 7-9). Further explorations for reaction solvents displayed that DCE was the best choice for this transformation (entries 10,11). In addition, we attempted some complex additives with HOAc, CsF, or $\mathrm{Zn}(\mathrm{OAc})_{2}$, respectively, but they led to a slightly decreasing yield (entries 12-14). Similarly, reducing the reaction temperature to $80^{\circ} \mathrm{C}$ or $60{ }^{\circ} \mathrm{C}$ was also detrimental to this transformation (entries 15,16 ).

Table 1. Optimization of Reaction Conditions ${ }^{a}$.
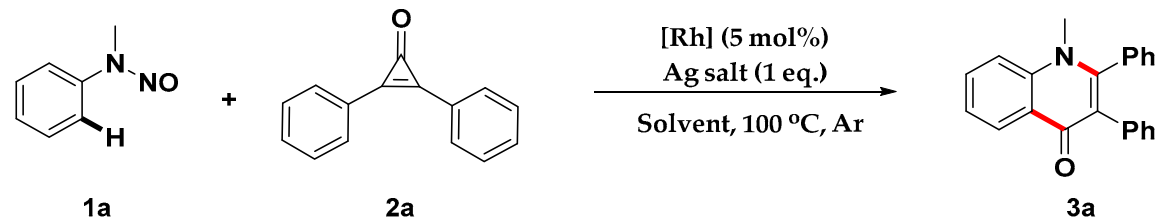

\begin{tabular}{|c|c|c|c|c|}
\hline Entry & [Rh] & {$[\mathrm{Ag}]$} & Solvent & Yield $(\%)^{b}$ \\
\hline 1 & {$\left[\mathrm{Cp}^{*} \mathrm{RhCl}_{2}\right]_{2}$} & $\mathrm{AgBF}_{4}$ & DCE & 13 \\
\hline 2 & $\mathrm{Rh}\left(\mathrm{PPh}_{3}\right)_{3} \mathrm{Cl}$ & $\mathrm{AgBF}_{4}$ & DCE & 0 \\
\hline 3 & $\mathrm{Rh}(\mathrm{COD})_{2}\left(\mathrm{BF}_{4}\right)$ & $\mathrm{AgBF}_{4}$ & DCE & 0 \\
\hline 4 & 1 & $\mathrm{AgBF}_{4}$ & DCE & 0 \\
\hline $5^{c}$ & {$\left[\mathrm{Cp}^{*} \mathrm{RhCl}_{2}\right]_{2}$} & $\mathrm{AgBF}_{4}$ & DCE & 49 \\
\hline $6^{d}$ & {$\left[\mathrm{Cp}^{*} \mathrm{RhCl}_{2}\right]_{2}$} & $\mathrm{AgBF}_{4}$ & DCE & $72(70)^{j}$ \\
\hline $7^{d}$ & {$\left[\mathrm{Cp}^{*} \mathrm{RhCl}_{2}\right]_{2}$} & $\mathrm{AgSbF}_{6}$ & DCE & 40 \\
\hline $8^{d}$ & {$\left[\mathrm{Cp} * \mathrm{RhCl}_{2}\right]_{2}$} & AgOTf & DCE & 23 \\
\hline $9^{d}$ & {$\left[\mathrm{Cp}^{*} \mathrm{RhCl}_{2}\right]_{2}$} & 1 & DCE & 0 \\
\hline $10^{\mathrm{d}}$ & {$\left[\mathrm{Cp}^{*} \mathrm{RhCl}_{2}\right]_{2}$} & $\mathrm{AgBF}_{4}$ & THF & 69 \\
\hline $11^{\mathrm{d}}$ & {$\left[\mathrm{Cp}^{*} \mathrm{RhCl}_{2}\right]_{2}$} & $\mathrm{AgBF}_{4}$ & Acetone & 66 \\
\hline $12^{\mathrm{d}, \mathrm{e}}$ & {$\left[\mathrm{Cp}^{*} \mathrm{RhCl}_{2}\right]_{2}$} & $\mathrm{AgBF}_{4}$ & DCE & 49 \\
\hline $13^{d, f}$ & {$\left[\mathrm{Cp}^{*} \mathrm{RhCl}_{2}\right]_{2}$} & $\mathrm{AgBF}_{4}$ & DCE & 49 \\
\hline $14^{\mathrm{d}, \mathrm{g}}$ & {$\left[\mathrm{Cp}^{*} \mathrm{RhCl}_{2}\right]_{2}$} & $\mathrm{AgBF}_{4}$ & DCE & 67 \\
\hline $15^{\mathrm{d}, \mathrm{h}}$ & {$\left[\mathrm{Cp}^{*} \mathrm{RhCl}_{2}\right]_{2}$} & $\mathrm{AgBF}_{4}$ & DCE & 57 \\
\hline $16^{\mathrm{d}, \mathrm{i}}$ & {$\left[\mathrm{Cp} * \mathrm{RhCl}_{2}\right]_{2}$} & $\mathrm{AgBF}_{4}$ & DCE & 0 \\
\hline
\end{tabular}

\footnotetext{
a Reaction conditions: 1a $(0.2 \mathrm{mmol}), \mathbf{2 a}(0.2 \mathrm{mmol})$, Ag salt $(0.2 \mathrm{mmol})$, solvent $(2 \mathrm{~mL})$, sealed tube under argon, 2 h. ${ }^{b}$ NMR yield using $\mathrm{CH}_{2} \mathrm{Br}_{2}$ as internal standard. ${ }^{\mathrm{c}}$ solvent $(5 \mathrm{~mL}) .{ }^{\mathrm{d}}$ solvent $(10 \mathrm{~mL}) .{ }^{\mathrm{e}} \mathrm{HOAc}(20 \mathrm{~mol} \%)$ was added. ${ }^{\mathrm{f}} \mathrm{CsF}(20 \mathrm{~mol} \%)$ was added. ${ }^{\mathrm{g}} \mathrm{Zn}(\mathrm{OAc})_{2}(20 \mathrm{~mol} \%)$ was added. ${ }^{\mathrm{h}}$ at $80^{\circ} \mathrm{C}^{\mathrm{i}}{ }^{\mathrm{i}}$ at $60^{\circ} \mathrm{C} .{ }^{\mathrm{j}}$ isolated yield. DCE: dichloroethane. THF: tetrahydrofuran.
}

With the optimized reaction conditions in hand, we firstly investigated the scope of $N$-nitrosoanilines, and the results indicated that this formal [3+3] annulation reaction could tolerate various substituents on both the aromatic ring $\left(R_{1}\right)$ and the nitrogen atom $\left(R_{2}\right)$ to generate diversified quinolin-4(1H)-one derivatives in moderate to good yields (Scheme 2). The introduction of 
electron-donating groups $\left(\mathrm{CH}_{3}\right.$ and $\left.\mathrm{OCH}_{3}\right)$ or electron-withdrawing groups $\left(\mathrm{COOMe}\right.$ and $\left.\mathrm{CF}_{3}\right)$ at the 4-position of aniline $\mathbf{1}$ was tolerant and had no influence on the yields ( $\mathbf{3 b}-\mathbf{3 h}$ ). Likewise, halogen-substituted anilines were also compatible in this catalytic system, giving the target compounds 3f-3h. When meta-substituted anilines were employed, the $\mathrm{C}-\mathrm{H}$ bond activation took place at the less sterically hindered position, irrespective of the electronic nature of the substituents, and both electron-donating and electron-withdrawing groups were converted smoothly into the desired products $3 \mathbf{i}$ and $3 \mathbf{j}$. Additionally, different $N$-substituents were explored, and the results showed that the substrates bearing alkyl and benzylic substituents could afford the desired products in moderate yields $(3 k-3 m)$.

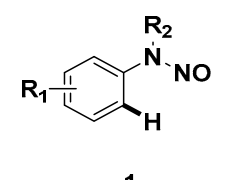

1<smiles>O=C(c1ccccc1)c1ccccc1</smiles>

$2 a$
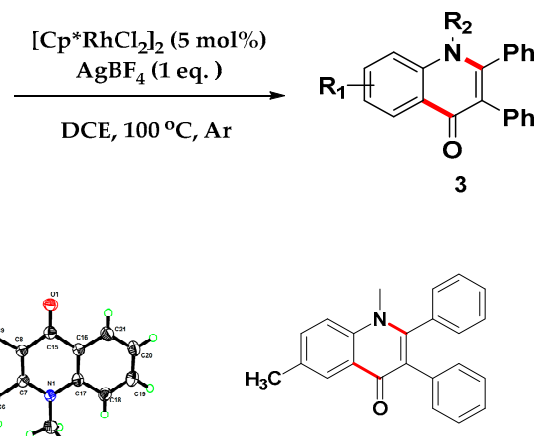

$3 b, 68 \%$<smiles>COc1ccc2c(c1)C(=O)C(c1ccccc1)C(c1ccccc1)N2C</smiles>

3c, $54 \%$<smiles>Cn1c(-c2ccccc2)c(-c2ccccc2)c(=O)c2cc(F)ccc21</smiles>

$3 f, 53 \%$

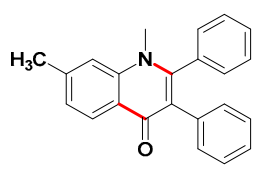

$3 \mathrm{i}, 63 \%$

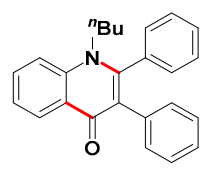

$31,54 \%$

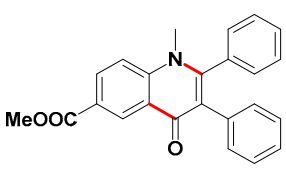

$3 d, 61 \%$

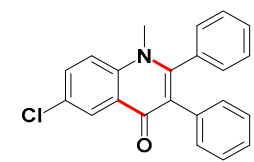

$3 g, 54 \%$

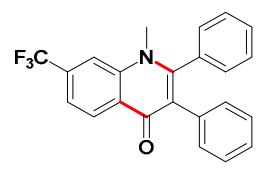

$3 \mathrm{j}, 46 \%$

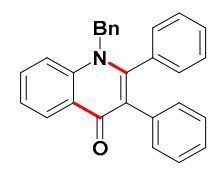

$3 \mathrm{~m}, 50 \%$

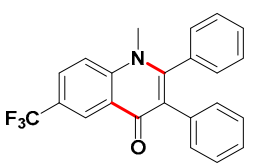

$3 e, 61 \%$<smiles></smiles>

$3 \mathrm{~h}, 60 \%$

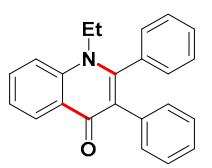

$3 k, 54 \%$

Scheme 2. Scope of $N$-nitrosoanilines. Reaction conditions: 1 (0.2 mmol), $2(0.4 \mathrm{mmol}), \mathrm{AgBF}_{4}$ $(0.2 \mathrm{mmol}), \mathrm{DCE}(10 \mathrm{~mL})$, sealed tube under argon, $2 \mathrm{~h}$. The percentage represents isolated yield.

Next, the scope of cyclopropenones was further tested (Scheme 3), and the results demonstrated that different cyclopropenones could proceed smoothly to provide the corresponding products. The cyclopropenones bearing an electron-donating group at the para position of the phenyl group, such as methyl, tert-butyl, and methoxyl, were well tolerated under standard conditions, giving the desired products in moderate to good yields $(\mathbf{4 a}-\mathbf{4 i})$, regardless of whether electron-donating groups or electron-withdrawing groups were equipped into the $N$-aniline ring. Moreover, halogen-substituted 
phenyl groups could also be smoothly transformed into the corresponding products in moderate to good yields $(4 \mathbf{j}-4 \mathbf{n})$.

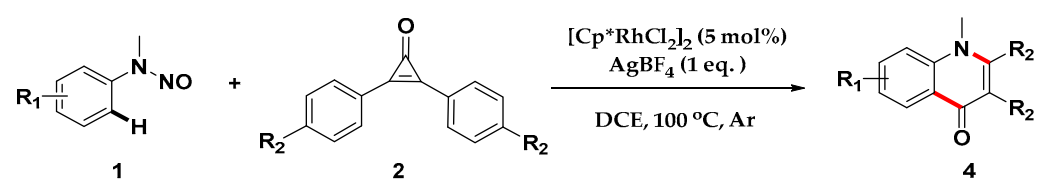

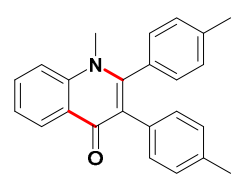

$4 a, 44 \%$

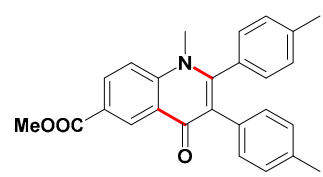

4d, $58 \%$

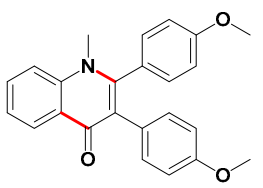

$4 g, 36 \%$

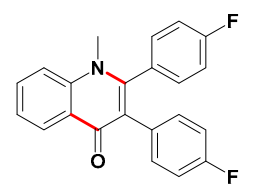

$4 \mathrm{j}, \mathbf{4 7 \%}$<smiles>Cc1ccc2c(c1)c(=O)c(-c1ccc(Cl)cc1)c(-c1ccc(Cl)cc1)n2C</smiles>

$4 \mathrm{~m}, 68 \%$

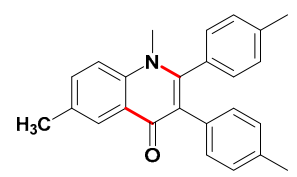

$4 b, 65 \%$<smiles>Cn1c(-c2ccc(C(C)(C)C)cc2)c(-c2ccc(C(C)(C)C)cc2)c(=O)c2cc(Cl)ccc21</smiles>

$4 e, 31 \%$<smiles>COc1ccc(-c2c(-c3ccc(OC)cc3)n(C)c3ccc(Cl)cc3c2=O)cc1</smiles>

4h, $52 \%$<smiles>Cn1c(-c2ccc(F)cc2)c(-c2ccc(F)cc2)c(=O)c2cc(Cl)ccc21</smiles>

$4 k, 68 \%$<smiles>Cc1ccc(-c2c(-c3ccc(C)cc3)n(C)c3ccc(Cl)cc3c2=O)cc1</smiles>

$4 c, 53 \%$<smiles>CC(=O)c1ccc2c(c1)c(=O)c(-c1ccc(C(C)(C)C)cc1)c(-c1ccc(C(C)(C)C)cc1)n2C</smiles>

4f, $28 \%$<smiles>COc1ccc(-c2c(-c3ccc(OC)cc3)n(C)c3ccc(C(C)=O)cc3c2=O)cc1</smiles>

$4 i, 41 \%$<smiles>CC(=O)c1ccc2c(c1)c(=O)c(-c1ccc(F)cc1)c(-c1ccc(F)cc1)n2C</smiles>

$41,59 \%$

Scheme 3. Scope of cyclopropenones. Reaction conditions: 1 (0.2 mmol), 2 (0.3 mmol), AgBF $(0.2 \mathrm{mmol}), \mathrm{DCE}(10 \mathrm{~mL})$, sealed tube under argon, $2 \mathrm{~h}$. The percentage represents isolated yield.

Intrigued by the privileged heterocyclic product derived from our strategy, we have further explored the gram-scale preparation of this transformation, its synthetic utility, and the late-stage functionalization for some important privileged scaffolds. As shown in Scheme 4, the redox-neutral [3+3] annulation could be carried out on a gram scale to produce $3 \mathbf{a}$ in a $57 \%$ yield (Scheme $4 a$ ). The synthetic utility of the obtained quinolin- $4(1 \mathrm{H})$-one derivatives has been demonstrated by the following transformations into potentially bioactive molecules (Scheme 4b). Treatment of 3a with Lawesson's reagent furnished thioketone 5 in a $95 \%$ yield, which could be further converted into thio-substituted product 6 in the presence of ethyl bromoacetate with a high yield. More interestingly, this strategy 
could also be used in the late-stage functionalization for tetrahydroquinoline privileged scaffolds to afford highly fused heterocyclic scaffolds, $\mathbf{3 n}-\mathbf{3 p}$ (Scheme $4 \mathrm{c}$ ).

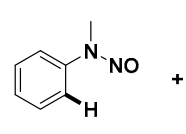

$1 \mathrm{a}(2 \mathrm{mmol})$

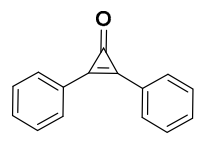

2a

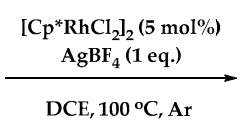

DCE, $100^{\circ} \mathrm{C}, \mathrm{Ar}$

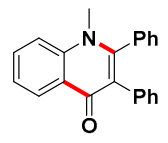

$3 a(57 \%)$ (a)

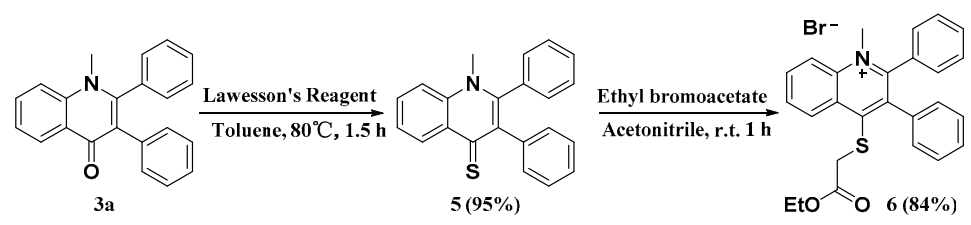<smiles>[R]c1ccc2c(c1)CCCN2[N+](=O)[O-]</smiles>

$1 n-p$<smiles></smiles>

$2 a$

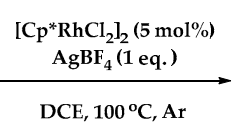

DCE, $100^{\circ} \mathrm{C}, \mathrm{Ar}$

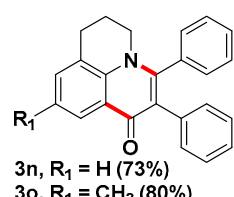

$30, \mathrm{R}_{1}=\mathrm{CH}_{3}(80 \%)$

$30, R_{1}=\mathrm{CH}_{3}(80 \%)$
$3 \mathrm{p}, \mathrm{R}_{1}=\mathrm{COOMe}(85 \%)$

Scheme 4. Gram experiment and derivatization of coupled product. (a) Gram-scale experiment.

(b) Derivatization of final product. (c) Late-stage functionalization for tetrahydroquinolines.

To understand the reaction mechanism, control experiments were carried out (Scheme 5). Firstly, the hydrogen-deuterium (H/D) exchange experiment was conducted to gain insight into the $\mathrm{C}-\mathrm{H}$ cleavage step. No deuterated $N$-nitrosoaniline was observed after treating with $\mathrm{CD}_{3} \mathrm{OD}$, indicating that rhodium-mediated C-H bond cleavage is irreversible (Scheme 5a). D5-1a and 1a were then subjected to the standard conditions, and the kinetic isotope effect (KIE) was measured. The value of $\mathrm{kH} / \mathrm{kD}$ is 1.7 , implying that the $\mathrm{C}-\mathrm{H}$ bond cleavage was the rate-determining step in the transformation (Scheme 5b) [43]. Furthermore, to probe the electronic preference, an intermolecular competition experiment was carried out, and the result suggested that the electron-rich substrate, $\mathbf{1} \mathbf{b}$, reacted at a higher rate (Scheme 5c).

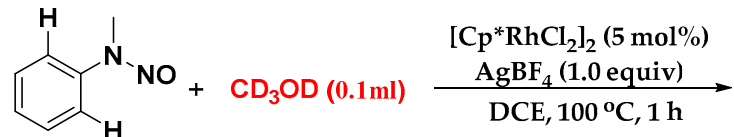

1a<smiles>CN(c1ccccc1)c1ccccc1</smiles>

$$
\text { d5-1a }
$$<smiles>Cc1ccc(N(C)N)cc1</smiles>

$1 b$ $\mathrm{AgBF}_{4}$ (1.0 equiv)

$\left[\mathrm{Cp}^{*} \mathrm{RhCl}_{2} \mathrm{I}_{2}(5 \mathrm{~mol} \%)\right.$ $\operatorname{AgBF}_{4}(1.0$ equiv)
2a $(1.5$ equiv)
DCE, $100^{\circ} \mathrm{C}, 10 \mathrm{~min}$ $K_{\mathrm{H}} / K_{\mathrm{D}}=1.70$

1a<smiles>CN([O-])c1c([18OH])cccc1[18OH]</smiles>

d2-1a (<5\%)<smiles></smiles><smiles>[R]c1ccc2c(c1)c(=O)c(-c1ccccc1)c(-c1ccccc1)n2C</smiles> 
On the basis of these results and literature precedents [20,21], in order to gain insight into this reaction mechanism, the mechanism of the coupling of $N$-nitrosoaniline with cyclopropenone is proposed in Scheme 6. A cationic Rh(III) species can easily undergo ortho C-H insertion of $N$-nitrosoaniline 1a to afford intermediate I. Then, intermediate I can be saturated by cyclopropenone coordination and subsequently undergo migratory insertion of the Rh-C bond into the carbonyl group of cyclopropenone $\mathbf{2 a}$ to afford the alkoxide intermediate II, which is followed by $\beta$-carbon elimination to afford the Rh(III) alkenyl intermediate III. Finally, intermediate III undergoes a direct cyclization pathway to yield the six-membered ring product, 3a, releasing $\mathrm{HNO}$ with the regeneration of the Rh(III) catalyst.

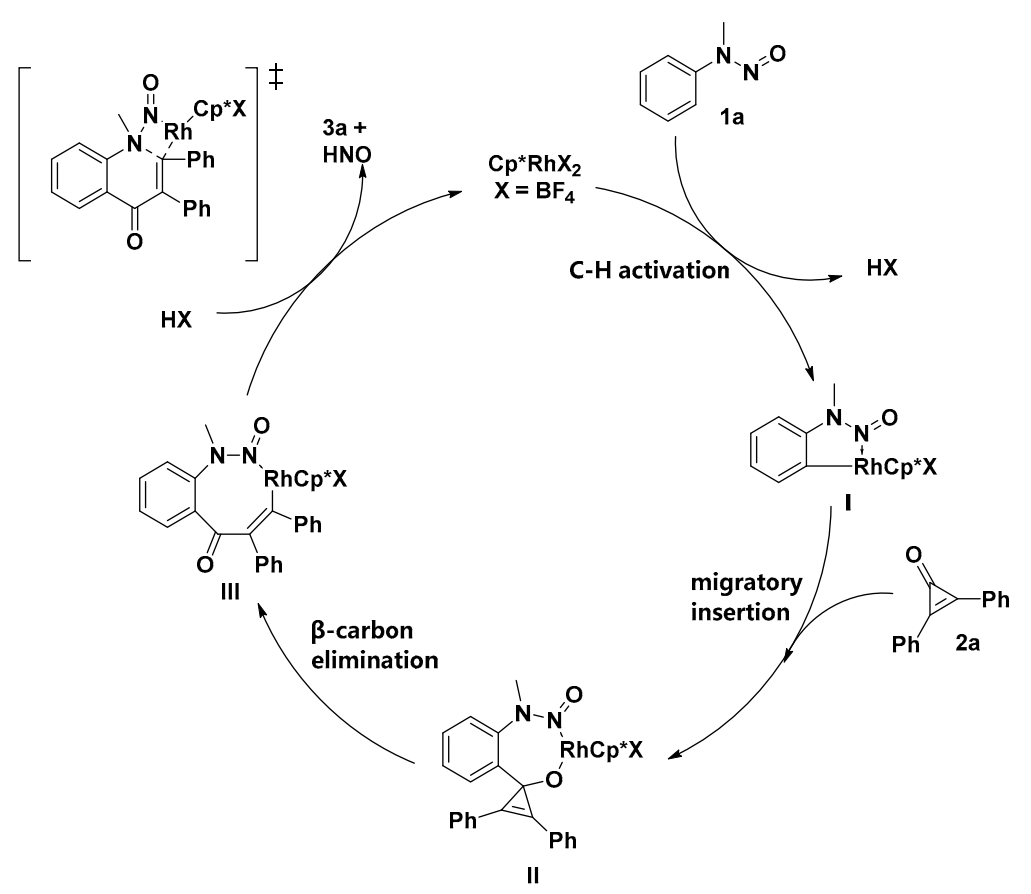

Scheme 6. Proposed mechanism.

\section{Materials and Methods}

\subsection{General Information}

Unless otherwise noted, the reagents (chemicals) were purchased from commercial sources and used without further purification. Water was deionized before being used. Analytical thin layer chromatography (TLC) was HSGF 254 (0.15-0.2 mm thickness). Compound spots were visualized by UV light (254 nm). Column chromatography was performed on silica gel FCP 300-400. NMR spectra were run on a 400 or $500 \mathrm{MHz}$ instrument. Chemical shifts were reported in parts per million (ppm, $\delta$ ) downfield from tetramethylsilane. Proton coupling patterns are described as singlet (s), doublet (d), triplet $(\mathrm{t})$, quartet $(\mathrm{q})$, multiplet $(\mathrm{m})$, and broad (br). Low- and high-resolution mass spectra (LRMS and HRMS) were measured on a spectrometer. $N$-nitrosoanilines $\mathbf{1}$ and cyclopropenones $\mathbf{2}$ were prepared according to the previous literature [23,44-46].

\subsection{General Procedures for Rhodium(III)-Catalyzed Redox-Neutral [3+3] Annulation of N-Nitrosoanilines with Cyclopropenones (3 and 4)}

To a $35 \mathrm{~mL}$ Schlenk tube was sequentially added $N$-nitrosoanilines $\mathbf{1}(0.2 \mathrm{mmol})$, cyclopropenone $2(0.4 \mathrm{mmol}$ for product $3,0.3 \mathrm{mmol}$ for product 4$)$, catalyst ( $5 \mathrm{~mol} \%), \mathrm{Ag}$ salt $(0.2 \mathrm{mmol})$, and solvent $(10 \mathrm{~mL})$. The reaction was sealed under argon and stirred at $100{ }^{\circ} \mathrm{C}$ for $2 \mathrm{~h}$. After the reaction was completed (detected by TLC), solvent was removed under reduced pressure, and the crude mixture was purified by flash column chromatography on silica gel with a PE/EA $(4 / 1, v / v)$ solvent system to 
afford the final product, 3, and with a $\mathrm{CH}_{2} \mathrm{Cl}_{2} / \mathrm{CH}_{3} \mathrm{OH}(50 / 1, v / v)$ solvent system to afford the final product, 4 .

\subsection{General Procedures for 1-Methyl-2,3-diphenylquinoline-4(1H)-thione (5)}

To a solution of $3 \mathbf{a}(50 \mathrm{mg}, 0.16 \mathrm{mmol})$ in $20 \mathrm{~mL}$ toluene was added Lawesson's reagent $(64 \mathrm{mg}$, $0.16 \mathrm{mmol}$ ), and the reaction was stirred at $80{ }^{\circ} \mathrm{C}$ for $1.5 \mathrm{~h}$. After the reaction was completed, the mixture was filtered, and the precipitate was washed with cold ethanol to afford the compound, 5, as a brown solid in a $95 \%$ yield.

\subsection{General Procedures for 4-((2-Ethoxy-2-oxoethyl)thio)-1-methyl-2,3-diphenylquinolin-1-ium (6)}

A $50 \mathrm{~mL}$ reaction flask was charged with acetonitrile and compound 5 (20 mg, $0.06 \mathrm{mmol})$, and ethyl bromoacetate (1.2 equiv) was added for $1 \mathrm{~h}$ at room temperature. After the reaction was completed, the solvent was removed, and the residue was purified by flash column chromatography on silica gel eluting with methanol to afford the compound, 6 , in a $84 \%$ yield.

\subsection{Analytical Characterization Data of Products}

1-Methyl-2,3-diphenylquinolin-4(1H)-one (3a). Light yellow solid (70\%). m.p. 218.3-218. ${ }^{\circ} \mathrm{C} ;{ }^{1} \mathrm{H}$ NMR $(500 \mathrm{MHz}$, Chloroform- $d$ ) $\delta 8.59(\mathrm{dd}, J=8.0,1.7 \mathrm{~Hz}, 1 \mathrm{H}), 7.73(\mathrm{ddd}, J=8.7,7.0,1.7 \mathrm{~Hz}, 1 \mathrm{H}), 7.57(\mathrm{~d}$, $J=8.6 \mathrm{~Hz}, 1 \mathrm{H}), 7.46-7.41(\mathrm{~m}, 1 \mathrm{H}), 7.30-7.26(\mathrm{~m}, 3 \mathrm{H}), 7.18-7.14(\mathrm{~m}, 2 \mathrm{H}), 7.13-7.09(\mathrm{~m}, 2 \mathrm{H}), 7.07-7.02(\mathrm{~m}$, $3 \mathrm{H}), 3.55$ (s, 3H); ${ }^{13} \mathrm{C}$ NMR $(125 \mathrm{MHz}$, Chloroform-d) $\delta 176.4,152.2,141.6,135.9,135.2,132.4,131.5$, 129.7, 128.8, 128.4, 127.6, 127.6, 126.8, 126.2, 124.5, 123.7, 115.9, 37.8. IR $\left(\mathrm{KBr}, \mathrm{cm}^{-1}\right): 3056,1616,1531$, $1482,1321,1068,754$. HRMS (Electrospray ionization ESI) $m / z[\mathrm{M}+\mathrm{H}]^{+}$calcd. for $\mathrm{C}_{22} \mathrm{H}_{18} \mathrm{NO}$ : 312.1383, found: 312.1384 .

1,6-Dimethyl-2,3-diphenylquinolin-4(1H)-one (3b). Light yellow solid (68\%). m.p. $187.5-187.8{ }^{\circ} \mathrm{C} ;{ }^{1} \mathrm{H}$ NMR (400 MHz, Chloroform- $d$ ) $\delta 8.39-8.35(\mathrm{~m}, 1 \mathrm{H}), 7.55(\mathrm{dd}, J=8.7,2.2 \mathrm{~Hz}, 1 \mathrm{H}), 7.48(\mathrm{~d}, J=8.8$ $\mathrm{Hz}, 1 \mathrm{H}), 7.29-7.26(\mathrm{~m}, 3 \mathrm{H}), 7.17-7.08(\mathrm{~m}, 4 \mathrm{H}), 7.06-7.01(\mathrm{~m}, 3 \mathrm{H}), 3.53(\mathrm{~s}, 3 \mathrm{H}), 2.52(\mathrm{~s}, 3 \mathrm{H}) ;{ }^{13} \mathrm{C} \mathrm{NMR}$ (125 MHz, Chloroform-d) $\delta 176.3,151.9,139.7,136.2,135.3,133.8,133.6,131.6,129.8,128.8,128.4,127.6$, $126.9,126.7,126.2,124.2,115.8,37.7,21.1$. HRMS (ESI) $m / z[\mathrm{M}+\mathrm{H}]^{+}$calcd. for $\mathrm{C}_{23} \mathrm{H}_{20} \mathrm{NO}$ : 326.1539, found: 326.1539 .

6-Methoxy-1-methyl-2,3-diphenylquinolin-4(1H)-one (3c). Yellow solid (54\%). m.p. $212.4-212.7{ }^{\circ} \mathrm{C} ;{ }^{1} \mathrm{H}$ NMR $(400 \mathrm{MHz}$, Chloroform- $d) \delta 8.00(\mathrm{~d}, J=3.1 \mathrm{~Hz}, 1 \mathrm{H}), 7.54(\mathrm{~d}, J=9.3 \mathrm{~Hz}, 1 \mathrm{H}), 7.37-7.35(\mathrm{~m}, 1 \mathrm{H})$, 7.29-7.28 (m, 3H), 7.18-7.08 (m, 4H), 7.07-7.01 (m, 3H), 3.95 (s, 3H), $3.56(\mathrm{~s}, 3 \mathrm{H}) ;{ }^{13} \mathrm{C}$ NMR $(125 \mathrm{MHz}$, Chloroform- $d$ ) $\delta 175.9,156.7,151.8,136.5,136.4,135.4,131.8,130.0,129.1,128.7,128.1,127.8,126.5$, $123.8,123.3,117.9,106.7,56.2,38.2$. HRMS (ESI) $m / z[\mathrm{M}+\mathrm{H}]^{+}$calcd. for $\mathrm{C}_{23} \mathrm{H}_{20} \mathrm{NO}_{2}: 342.1489$, found: 342.1495 .

6-Methoxycarbonyl-1-methyl-2,3-diphenylquinoline-4(1H)-one (3d). Light yellow solid (61\%). m.p. 236.7-237.2 ${ }^{\circ} \mathrm{C} ;{ }^{1} \mathrm{H}$ NMR (600 MHz, DMSO-d6) $\delta 8.86(\mathrm{~d}, \mathrm{~J}=2.3 \mathrm{~Hz}, 1 \mathrm{H}), 8.27(\mathrm{dd}, \mathrm{J}=9.0,2.2$ $\mathrm{Hz}, 1 \mathrm{H}), 7.92(\mathrm{~d}, J=9.1 \mathrm{~Hz}, 1 \mathrm{H}), 7.23-7.06(\mathrm{~m}, 4 \mathrm{H}), 6.94-6.91(\mathrm{~m}, 2 \mathrm{H}), 6.88-6.85(\mathrm{~m}, 2 \mathrm{H}), 3.92(\mathrm{~s}, 3 \mathrm{H})$, $3.47(\mathrm{~s}, 3 \mathrm{H}), 2.27(\mathrm{~s}, 3 \mathrm{H}), 2.19(\mathrm{~s}, 3 \mathrm{H}) ;{ }^{13} \mathrm{C}$ NMR $(125 \mathrm{MHz}$, Chloroform- $d) \delta 176.3,166.7,152.6,144.2$, 135.3, 134.8, 132.8, 131.3, 130.1, 129.6, 129.1, 128.6, 127.7, 126.5, 126.2, 125.6, 125.3, 116.2, 52.4, 38.1 . HRMS (ESI) $m / z$ [M + H] $]^{+}$calcd. for $\mathrm{C}_{24} \mathrm{H}_{20} \mathrm{NO}_{3}$ : 370.1438, found: 370.1430 .

6-(Trifluoromethyl)-1-methyl-2,3-diphenyl-quinolin-4(1H)-one (3e). Light yellow solid (61\%). m.p. 226.7-227.0 ${ }^{\circ} \mathrm{C} ;{ }^{1} \mathrm{H}$ NMR $(400 \mathrm{MHz}$, Chloroform- $d) \delta 8.86(\mathrm{~d}, J=2.2 \mathrm{~Hz}, 1 \mathrm{H}), 7.95-7.90(\mathrm{~m}, 1 \mathrm{H})$, $7.67(\mathrm{~d}, J=9.0 \mathrm{~Hz}, 1 \mathrm{H}), 7.33-7.27(\mathrm{~m}, 3 \mathrm{H}), 7.18-7.06(\mathrm{~m}, 5 \mathrm{H}), 7.06-6.98(\mathrm{~m}, 2 \mathrm{H}), 3.57(\mathrm{~s}, 3 \mathrm{H}) .{ }^{13} \mathrm{C} \mathrm{NMR}$ $(125 \mathrm{MHz}$, Chloroform- $d$ ) $\delta 175.9,152.9,143.3,135.2,134.6,131.3,129.6,129.2,128.6,128.5(\mathrm{q}, J=3.0 \mathrm{~Hz})$ $127.7,126.6,126.3,125.9,125.6(\mathrm{q}, J=3.0 \mathrm{~Hz}), 125.1,123.3(\mathrm{q}, J=271.8 \mathrm{~Hz}), 116.9,38.0 .{ }^{19} \mathrm{~F}$ NMR (471 MHz, Chloroform- $d$ ) $\delta$-61.9. HRMS (ESI) $m / z$ [M + H]+ calcd. for $\mathrm{C}_{23} \mathrm{H}_{17} \mathrm{~F}_{3} \mathrm{NO}$ : 380.1257, found 380.1251 . 
6-Fluoro-1-methyl-2,3-diphenylquinolin-4(1H)-one (3f). White solid (53\%). m.p. $213.5-213.8{ }^{\circ} \mathrm{C} ;{ }^{1} \mathrm{H}$ NMR $(500 \mathrm{MHz}$, Chloroform- $d) \delta 8.22(\mathrm{dd}, J=8.9,3.1 \mathrm{~Hz}, 1 \mathrm{H}), 7.58(\mathrm{dd}, J=9.3,4.1 \mathrm{~Hz}, 1 \mathrm{H}), 7.46(\mathrm{ddd}, J=9.3$, 7.5, 3.1 Hz, 1H), 7.30-7.27 (m, 3H), 7.17-7.09 (m, 4H), 7.08-7.00 (m, 3H), $3.56(\mathrm{~s}, 3 \mathrm{H}) ;{ }^{13} \mathrm{C} \mathrm{NMR}(125$ MHz, Chloroform-d) $\delta 175.6,159.4(\mathrm{~d}, J=243.9 \mathrm{~Hz}), 152.3,138.1,135.7,134.9,131.4,129.7,129.0,128.5$, $128.3(\mathrm{~d}, J=6.8 \mathrm{~Hz}), 127.7,126.4,123.9,120.9(\mathrm{~d}, J=24.8 \mathrm{~Hz}), 118.2(\mathrm{~d}, J=7.6 \mathrm{~Hz}), 112.1(\mathrm{~d}, J=22.3 \mathrm{~Hz})$, 38.1. ${ }^{19} \mathrm{~F}$ NMR (471 MHz, Chloroform- $d$ ) $\delta$-118.0. HRMS (ESI) $m / z[\mathrm{M}+\mathrm{H}]{ }^{+}$calcd. for $\mathrm{C}_{22} \mathrm{H}_{17} \mathrm{FNO}$ : 330.1289, found: 330.1288 .

6-Chloro-1-methyl-2,3-diphenylquinolin-4(1H)-one (3g). Light yellow solid (54\%). m.p. 248.4-248.9 ${ }^{\circ} \mathrm{C}$; ${ }^{1} \mathrm{H}$ NMR (500 MHz, Chloroform- $\left.d\right) \delta 8.53(\mathrm{~d}, J=2.5 \mathrm{~Hz}, 1 \mathrm{H}), 7.66(\mathrm{dd}, J=9.1,2.6 \mathrm{~Hz}, 1 \mathrm{H}), 7.52(\mathrm{~d}$, $J=9.1 \mathrm{~Hz}, 1 \mathrm{H}), 7.30-7.27(\mathrm{~m}, 3 \mathrm{H}), 7.18-7.09(\mathrm{~m}, 4 \mathrm{H}), 7.09-6.98(\mathrm{~m}, 3 \mathrm{H}), 3.54(\mathrm{~s}, 3 \mathrm{H}) ;{ }^{13} \mathrm{C}$ NMR $(125$ MHz, Chloroform-d) $\delta 175.3,152.4,140.0,135.5,134.9,132.6,131.4,130.0,129.7,129.0,128.5,127.8,127.7$, $126.9,126.5,124.8,117.7,38.0$. HRMS (ESI) $\mathrm{m} / z$ [M + H] $]^{+}$calcd. for $\mathrm{C}_{22} \mathrm{H}_{17} \mathrm{ClNO}$ : 346.0993, found: 346.0996.

6-Bromo-1-methyl-2,3-diphenylquinolin-4(1H)-one (3h). Light yellow solid $(60 \%)$. m.p. $243 \cdot 1-244.2{ }^{\circ} \mathrm{C} ;{ }^{1} \mathrm{H}$ NMR (500 MHz, Chloroform-d) $\delta 8.69(\mathrm{~d}, J=2.5 \mathrm{~Hz}, 1 \mathrm{H}), 7.79(\mathrm{dd}, J=9.0,2.5 \mathrm{~Hz}, 1 \mathrm{H}), 7.45(\mathrm{~d}, J=$ $9.1 \mathrm{~Hz}, 1 \mathrm{H}), 7.30-7.28(\mathrm{~m}, 3 \mathrm{H}), 7.16-7.09(\mathrm{~m}, 4 \mathrm{H}), 7.08-6.99(\mathrm{~m}, 3 \mathrm{H}), 3.53(\mathrm{~s}, 3 \mathrm{H}) ;{ }^{13} \mathrm{C} \mathrm{NMR}(125 \mathrm{MHz}$, Chloroform- $d$ ) $\delta 175.2,152.4,140.4,135.5,135.3,134.8,131.4,130.1,129.7,129.0,128.5,128.2,127.7,126.5$, 125.0, 117.9, 117.6, 37.9. HRMS (ESI) $m / z[\mathrm{M}+\mathrm{H}]^{+}$calcd. for $\mathrm{C}_{22} \mathrm{H}_{17} \mathrm{Br} \mathrm{NO}$ : 390.0488, found: 390.0482 .

1,7-Dimethyl-2,3-diphenylquinolin-4(1H)-one (3i). Light yellow solid (63\%). m.p. $286.1-287.6{ }^{\circ} \mathrm{C} ;{ }^{1} \mathrm{H}$ NMR (500 MHz, Chloroform- $d) \delta 8.46(\mathrm{~d}, J=8.2 \mathrm{~Hz}, 1 \mathrm{H}), 7.35(\mathrm{~s}, 1 \mathrm{H}), 7.30-7.26(\mathrm{~m}, 4 \mathrm{H}), 7.16-7.14(\mathrm{~m}$, 2H), 7.12-7.08 (m, 2H), 7.06-7.01 (m, 3H), $3.52(\mathrm{~s}, 3 \mathrm{H}), 2.56(\mathrm{~s}, 3 \mathrm{H}) ;{ }^{13} \mathrm{C}$ NMR (125 MHz, Chloroform- $d$ ) $\delta 176.3,151.9,143.1,141.8,136.0,135.3,131.6,129.8,128.8,128.4,127.5,127.4,126.2,125.4,124.8,124.3$, 115.6, 37.7, 22.5. HRMS (ESI) $m / z$ [M + H] $]^{+}$calcd. for $\mathrm{C}_{23} \mathrm{H}_{20} \mathrm{NO}$ : 326.1539, found: 326.1546 .

1-Methyl-2,3-diphenyl-7-(trifluoromethyl)quinolin-4(1H)-one (3j). Light yellow solid (46\%). m.p. 221.4-222.7 ${ }^{\circ} \mathrm{C} ;{ }^{1} \mathrm{H}$ NMR (500 MHz, Chloroform- $d$ ) $\delta 8.69(\mathrm{~d}, J=8.3 \mathrm{~Hz}, 1 \mathrm{H}), 7.84(\mathrm{~s}, 1 \mathrm{H}), 7.65$ $(\mathrm{dd}, J=8.4,1.4 \mathrm{~Hz}, 1 \mathrm{H}), 7.32-7.28(\mathrm{~m}, 3 \mathrm{H}), 7.18-7.11(\mathrm{~m}, 4 \mathrm{H}), 7.09-7.00(\mathrm{~m}, 3 \mathrm{H}), 3.59(\mathrm{~s}, 3 \mathrm{H}) ;{ }^{13} \mathrm{C} \mathrm{NMR}$ (125 MHz, Chloroform- $d$ ) $\delta$ 175.8, 153.1, 141.2, 135.3, 134.7, 134.0 (q, $J=32.5 \mathrm{~Hz}), 131.3,129.6,129.2$, 129.0, 128.6, 127.7, 126.6, 125.6, 124.0 (q, $J=262.5 \mathrm{~Hz}), 119.7$ (q, $J=3.2 \mathrm{~Hz}), 113.6$ (q, $J=4.2 \mathrm{~Hz}), 38.0$. ${ }^{19} \mathrm{~F}$ NMR (471 MHz, Chloroform- $d$ ) $\delta$-62.6. HRMS (ESI) $m / z[\mathrm{M}+\mathrm{H}]^{+}$calcd. for $\mathrm{C}_{23} \mathrm{H}_{17} \mathrm{~F}_{3} \mathrm{NO}$ : 380.1257, found: 380.1252 .

1-Ethyl-2,3-diphenylquinolin-4(1H)-one (3k). White solid (54\%). m.p. 247.4-247.9 ${ }^{\circ} \mathrm{C}^{1}{ }^{1} \mathrm{H} \mathrm{NMR}(400 \mathrm{MHz}$, Chloroform- $d) \delta 8.61(\mathrm{~d}, J=8.0 \mathrm{~Hz}, 1 \mathrm{H}), 7.75-7.68(\mathrm{~m}, 1 \mathrm{H}), 7.60(\mathrm{~d}, J=8.7 \mathrm{~Hz}, 1 \mathrm{H}), 7.43(\mathrm{t}, J=7.5$ $\mathrm{Hz}, 1 \mathrm{H}), 7.29-7.26(\mathrm{~m}, 3 \mathrm{H}), 7.22-7.17(\mathrm{~m}, 2 \mathrm{H}), 7.13-7.07(\mathrm{~m}, 2 \mathrm{H}), 7.06-7.00(\mathrm{~m}, 3 \mathrm{H}), 4.08(\mathrm{q}, J=7.0$ $\mathrm{Hz}, 2 \mathrm{H}), 1.30(\mathrm{t}, J=7.0 \mathrm{~Hz}, 3 \mathrm{H}) ;{ }^{13} \mathrm{C}$ NMR $(150 \mathrm{MHz}$, Chloroform-d $) \delta 176.5,152.2,140.3,136.2,135.2$, 132.6, 131.6, 129.6, 129.0, 128.6, 128.2, 127.8, 127.4, 126.5, 125.0, 123.8, 116.3, 43.9, 14.7. HRMS (ESI) $\mathrm{m} / \mathrm{z}$ $[\mathrm{M}+\mathrm{H}]^{+}$calcd. for $\mathrm{C}_{23} \mathrm{H}_{20} \mathrm{NO}$ : 326.1539 , found: 326.1532 .

1-Butyl-2,3-diphenylquinolin-4(1H)-one (31). White solid (54\%). m.p. 119.1-120.4 ${ }^{\circ} \mathrm{C} ;{ }^{1} \mathrm{H} \mathrm{NMR}(500 \mathrm{MHz}$, Chloroform- $d$ ) $\delta 8.61(\mathrm{dd}, J=8.1,1.7 \mathrm{~Hz}, 1 \mathrm{H}), 7.71(\mathrm{ddd}, J=8.7,7.0,1.7 \mathrm{~Hz}, 1 \mathrm{H}), 7.56(\mathrm{~d}, J=8.7 \mathrm{~Hz}$, $1 \mathrm{H}), 7.44-7.40(\mathrm{~m}, 1 \mathrm{H}), 7.30-7.27(\mathrm{~m}, 3 \mathrm{H}), 7.19-7.16(\mathrm{~m}, 2 \mathrm{H}), 7.10(\mathrm{dd}, J=8.3,6.5 \mathrm{~Hz}, 2 \mathrm{H}), 7.06-7.00(\mathrm{~m}$, $3 \mathrm{H}), 3.99-3.92(\mathrm{~m}, 2 \mathrm{H}), 1.70-1.68(\mathrm{~m}, 2 \mathrm{H}), 1.20-1.13(\mathrm{~m}, 2 \mathrm{H}), 0.77(\mathrm{t}, J=7.4 \mathrm{~Hz}, 3 \mathrm{H}) ;{ }^{13} \mathrm{C}$ NMR $(125$ $\mathrm{MHz}$, Chloroform- $d$ ) $\delta 176.2,152.1,140.3,136.0,135.0,132.3,131.5,129.5,128.8,128.3,127.9,127.6$, 127.1, 126.3, 124.7, 123.6, 116.2, 48.7, 30.9, 19.9, 13.5. HRMS (ESI) $m / z[\mathrm{M}+\mathrm{H}]^{+}$calcd. for $\mathrm{C}_{25} \mathrm{H}_{24} \mathrm{NO}$ : 354.1852, found: 354.1857 .

1-Benzyl-2,3-diphenylquinolin-4(1H)-one (3m). Light yellow solid (50\%). m.p. 74.3-75.6 ${ }^{\circ} \mathrm{C} ;{ }^{1} \mathrm{H}$ NMR $(500 \mathrm{MHz}$, Chloroform- $d) \delta 8.60(\mathrm{dd}, J=8.0,1.7 \mathrm{~Hz}, 1 \mathrm{H}), 7.55(\mathrm{ddd}, J=8.7,7.0,1.7 \mathrm{~Hz}, 1 \mathrm{H}), 7.41-7.35$ $(\mathrm{m}, 2 \mathrm{H}), 7.32-7.26(\mathrm{~m}, 3 \mathrm{H}), 7.19-7.02(\mathrm{~m}, 10 \mathrm{H}), 7.01-6.98(\mathrm{~m}, 2 \mathrm{H}), 5.25(\mathrm{~s}, 2 \mathrm{H}) ;{ }^{13} \mathrm{C}$ NMR $(125 \mathrm{MHz}$, Chloroform- $d$ ) $\delta 176.6,152.6,140.9,136.6,135.9,134.7,132.4,131.5,129.4,129.1,128.9,128.2,127.7$, 
127.6, 127.5 127.0, 126.3, 125.7, 124.9, 123.8, 117.2, 52.7. HRMS (ESI) $m / z[\mathrm{M}+\mathrm{H}]^{+}$calcd. for $\mathrm{C}_{28} \mathrm{H}_{22} \mathrm{NO}$ : 388.1696, found: 388.1698 .

2,3-Diphenyl-6,7-dihydro-1H,5H-pyrido [3,2,1-ij]quinolin-1-one (3n). Yellow solid (73\%). m.p. 280.2-281.4 ${ }^{\circ} \mathrm{C} ;{ }^{1} \mathrm{H}$ NMR $(500 \mathrm{MHz}$, Chloroform- $d) \delta 8.42(\mathrm{dd}, J=8.1,1.6 \mathrm{~Hz}, 1 \mathrm{H}), 7.45(\mathrm{dd}, J=7.1,1.5 \mathrm{~Hz}, 1 \mathrm{H})$, 7.33-7.24 (m, 4H), 7.18-7.13 (m, 2H), 7.13-7.07 (m, 2H), 7.06-7.00 (m, 3H), 3.82-3.75 (m, 2H), 3.08-3.04 (m, 2H), 2.12-2.05 (m, 2H); ${ }^{13} \mathrm{C}$ NMR (125 MHz, Chloroform-d) $\delta 176.3,151.3,138.2,136.0,134.9,131.5$, 131.5, 129.5, 128.7, 128.4, 127.5, 126.9, 126.8, 126.2, 125.5, 124.1, 123.2, 50.2, 28.0, 22.1. IR $\left(\mathrm{KBr}_{\mathrm{cm}}{ }^{-1}\right)$ : $3045,1616,1544,1484,1438,1307,1039,703$. HRMS (ESI) $m / z[\mathrm{M}+\mathrm{H}]^{+}$calcd. for $\mathrm{C}_{24} \mathrm{H}_{20} \mathrm{NO}: 338.1539$, found: 338.1536 .

9-Methyl-2,3-diphenyl-6,7-dihydro-1H,5H-pyrido[3,2,1-ij]quinolin-1-one (3o). Yellow solid (80\%). m.p. 251.2-252.3 ${ }^{\circ} \mathrm{C} ;{ }^{1} \mathrm{H}$ NMR $(500 \mathrm{MHz}$, Chloroform- $d$ ) $\delta 8.22-8.17(\mathrm{~m}, 1 \mathrm{H}), 7.30-7.26(\mathrm{~m}, 2 \mathrm{H}), 7.26-7.23(\mathrm{~m}$, 2H), 7.16-7.07 (m, 4H), 7.05-7.00 (m, 3H), 3.81-3.70 (m, 2H), 3.81-3.70 (m, 2H), 2.46 (s, 3H), 2.11-2.03 (m, 2H); ${ }^{13} \mathrm{C}$ NMR (125 MHz, Chloroform-d) $\delta 176.1,150.9,136.3,136.2,134.9,133.0,133.0,131.6,129.5$, 128.6, 128.4, 127.5, 126.8, 126.7, 126.1, 124.7, 123.8, 50.1, 27.9, 22.2, 21.1. IR (KBr, cm $\left.{ }^{-1}\right): 3056,1625,1536$, 1490, 1317, 1274, 1079, 711. HRMS (ESI) $\mathrm{m} / z$ [M + H] ${ }^{+}$calcd. for $\mathrm{C}_{25} \mathrm{H}_{22} \mathrm{NO}$ : 352.1696, found: 352.1697.

9-Methoxycarbonyl-2,3-diphenyl-6,7-dihydro-1H,5H-pyrido[3,2,1-ij]quinolin-1-one (3p). Yellow solid (85\%). m.p. 249.1-250.7 ${ }^{\circ} \mathrm{C} ;{ }^{1} \mathrm{H}$ NMR $(400 \mathrm{MHz}$, Chloroform- $d)$ 89.06-9.03 (m, 1H), 8.09-8.06 (m, 1H), 7.30-7.26 $(\mathrm{m}, 3 \mathrm{H}), 7.18-7.08(\mathrm{~m}, 4 \mathrm{H}), 7.08-6.99(\mathrm{~m}, 3 \mathrm{H}), 3.95(\mathrm{~s}, 3 \mathrm{H}), 3.82-3.75(\mathrm{~m}, 2 \mathrm{H}), 3.12-3.06(\mathrm{~m}, 2 \mathrm{H}), 2.14-2.05$ (m, 2H); ${ }^{13} \mathrm{C}$ NMR (125 MHz, Chloroform-d) $\delta 176.3,166.9,151.8,141.0,135.4,134.4,131.5,131.3,129.4$, 128.9, 128.6, 128.1, 127.6, 127.3, 126.5, 126.2, 125.2, 124.6, 52.3, 50.4, 27.9, 21.8. IR (KBr, $\left.\mathrm{cm}^{-1}\right): 3057,1714$, $1627,1498,1442,1301,1215,1072,701$. HRMS (ESI) $m / z[\mathrm{M}+\mathrm{H}]^{+}$calcd. for $\mathrm{C}_{26} \mathrm{H}_{22} \mathrm{NO}_{3}$ : 396.1594 , found: 396.1593 .

1-Methyl-2,3-di-p-tolylquinolin-4(1H)-one (4a). White solid (44\%). m.p. $186.3-186.7^{\circ} \mathrm{C} ;{ }^{1} \mathrm{H}$ NMR (500 $\mathrm{MHz}$, Chloroform- $d$ ) $\delta 8.59(\mathrm{dd}, J=8.0,1.7 \mathrm{~Hz}, 1 \mathrm{H}), 7.74-7.67(\mathrm{~m}, 1 \mathrm{H}), 7.56(\mathrm{~d}, J=8.6 \mathrm{~Hz}, 1 \mathrm{H}), 7.42$ $(\mathrm{ddd}, J=8.0,7.0,0.9 \mathrm{~Hz}, 1 \mathrm{H}), 7.09(\mathrm{~d}, J=7.8 \mathrm{~Hz}, 2 \mathrm{H}), 7.04(\mathrm{~d}, J=8.1 \mathrm{~Hz}, 2 \mathrm{H}), 6.94-6.91(\mathrm{~m}, 4 \mathrm{H}), 3.53(\mathrm{~s}$, $3 \mathrm{H}), 2.32(\mathrm{~s}, 3 \mathrm{H}), 2.22(\mathrm{~s}, 3 \mathrm{H}) ;{ }^{13} \mathrm{C}$ NMR $(125 \mathrm{MHz}$, Chloroform-d) $\delta 176.7,152.6,141.9,138.9,135.8$, 133.1, 132.6, 132.5, 131.5, 129.8, 129.4, 128.6, 127.9, 126.9, 124.7, 123.9, 116.1, 38.0, 21.7, 21.6. HRMS (ESI) $m / z[\mathrm{M}+\mathrm{H}]^{+}$calcd. for $\mathrm{C}_{24} \mathrm{H}_{22} \mathrm{NO}$ : 340.1696, found: 340.1694 .

1,6-Dimethyl-2,3-di-p-tolylquinolin-4(1H)-one (4b). White solid (65\%). m.p. 233.7-233.9 ${ }^{\circ} \mathrm{C} ;{ }^{1} \mathrm{H}$ NMR (500 MHz, Chloroform-d) $\delta 8.39-8.33(\mathrm{~m}, 1 \mathrm{H}), 7.52(\mathrm{dd}, J=8.8,2.1 \mathrm{~Hz}, 1 \mathrm{H}), 7.45(\mathrm{~d}, J=8.7 \mathrm{~Hz}, 1 \mathrm{H}), 7.08(\mathrm{~d}$, $J=7.9 \mathrm{~Hz}, 2 \mathrm{H}), 7.02(\mathrm{~d}, J=8.1 \mathrm{~Hz}, 2 \mathrm{H}), 6.92-6.90(\mathrm{~m}, 4 \mathrm{H}), 3.50(\mathrm{~s}, 3 \mathrm{H}), 2.50(\mathrm{~s}, 3 \mathrm{H}), 2.31(\mathrm{~s}, 3 \mathrm{H}), 2.22(\mathrm{~s}$, $3 \mathrm{H}) ;{ }^{13} \mathrm{C}$ NMR $(125 \mathrm{MHz}$, Chloroform-d) $\delta 176.1,151.7,139.4,138.3,135.2,133.4,133.1,132.8,132.2$, 131.1, 129.4, 128.8, 128.1, 126.7, 126.3, 123.9, 115.5, 37.4, 21.2, 21.1, 20.8. HRMS (ESI) $m / z$ [M + H] ${ }^{+}$calcd. for $\mathrm{C}_{25} \mathrm{H}_{24} \mathrm{NO}$ : 354.1852, found: 354.1850 .

6-Chloro-1-methyl-2,3-di-p-tolylquinolin-4(1H)-one (4c). Light yellow solid (53\%). m.p. 240.2-241.7 ${ }^{\circ} \mathrm{C}$; ${ }^{1} \mathrm{H}$ NMR (600 MHz, DMSO-d6) $\delta 8.19(\mathrm{~d}, J=2.5 \mathrm{~Hz}, 1 \mathrm{H}), 7.87(\mathrm{~d}, J=9.1 \mathrm{~Hz}, 1 \mathrm{H}), 7.83(\mathrm{dd}, J=9.1$, $2.6 \mathrm{~Hz}, 1 \mathrm{H}), 7.18-7.12(\mathrm{~m}, 4 \mathrm{H}), 6.93-6.89(\mathrm{~m}, 2 \mathrm{H}), 6.87-6.83(\mathrm{~m}, 2 \mathrm{H}), 3.45(\mathrm{~s}, 3 \mathrm{H}), 2.26(\mathrm{~s}, 3 \mathrm{H}), 2.18(\mathrm{~s}$, $3 \mathrm{H}) ;{ }^{13} \mathrm{C}$ NMR $(150 \mathrm{MHz}$, Chloroform- $d$ ) $\delta 175.7,152.7,140.2,139.1,136.0,132.7,132.6,132.2,131.3$, $129.9,129.7,129.4,128.6,127.9,126.9,124.9,118.0,38.1,21.7,21.6$. HRMS (ESI) $m / z[\mathrm{M}+\mathrm{H}]^{+}$calcd. for $\mathrm{C}_{24} \mathrm{H}_{21}$ CINO: 374.1306 , found: 374.1305 .

6-Methoxycarbonyl-1-methyl-2,3-di-p-tolylquinolin-4(1H)-one (4d). White solid (58\%). m.p. 238.1-239.4 ${ }^{\circ} \mathrm{C} ;{ }^{1} \mathrm{H}$ NMR (600 MHz, DMSO-d6) $\delta 8.86(\mathrm{~d}, J=2.3 \mathrm{~Hz}, 1 \mathrm{H}), 8.27(\mathrm{dd}, J=9.0,2.2 \mathrm{~Hz}, 1 \mathrm{H}), 7.92(\mathrm{~d}, J=$ $9.1 \mathrm{~Hz}, 1 \mathrm{H}), 7.23-7.06(\mathrm{~m}, 4 \mathrm{H}), 6.94-6.91(\mathrm{~m}, 2 \mathrm{H}), 6.88-6.85(\mathrm{~m}, 2 \mathrm{H}), 3.92(\mathrm{~s}, 3 \mathrm{H}), 3.47(\mathrm{~s}, 3 \mathrm{H}), 2.27(\mathrm{~s}$, 3H), 2.19 (s, 3H); ${ }^{13} \mathrm{C}$ NMR (150 MHz, Chloroform-d) $\delta$ 176.7, 167.0, 152.8, 144.4, 139.1, 136.1, 132.8, 132.5, 132.2, 131.3, 130.3, 129.7, 129.5, 128.7, 126.2, 125.7, 125.3, 116.4, 52.6, 38.2, 21.7, 21.6. HRMS (ESI) $m / z[\mathrm{M}+\mathrm{H}]^{+}$calcd. for $\mathrm{C}_{26} \mathrm{H}_{24} \mathrm{NO}_{3}: 398.1751$, found: 398.1744 . 
2,3-Bis(4-(tert-butyl)phenyl)-6-chloro-1-methylquinolin-4(1H)-one (4e). White solid (31\%). m.p. 296.0-297.6 ${ }^{\circ} \mathrm{C} ;{ }^{1} \mathrm{H}$ NMR (500 MHz, Chloroform-d) $\delta 8.56(\mathrm{~d}, J=2.5 \mathrm{~Hz}, 1 \mathrm{H}), 7.66(\mathrm{dd}, J=9.1,2.6 \mathrm{~Hz}, 1 \mathrm{H}), 7.54(\mathrm{~d}$, $J=9.0 \mathrm{~Hz}, 1 \mathrm{H}), 7.28(\mathrm{~d}, J=6.2 \mathrm{~Hz}, 2 \mathrm{H}), 7.10(\mathrm{~d}, J=8.0 \mathrm{~Hz}, 2 \mathrm{H}), 7.04(\mathrm{~d}, J=8.1 \mathrm{~Hz}, 2 \mathrm{H}), 6.91(\mathrm{~d}, J=8.0$ $\mathrm{Hz}, 2 \mathrm{H}), 3.62$ (s, 3H), 1.27 (s, 9H), 1.22 (s, 9H); ${ }^{13} \mathrm{C}$ NMR (150 MHz, Chloroform-d) $\delta 175.5,153.1,152.2$, $148.9,140.1,132.8,132.6,132.1,131.1,130.0,129.7,128.0,127.0,125.3,125.1,124.6,117.9,38.3,35.0,34.6$, 31.6, 31.5. HRMS (ESI) $m / z[\mathrm{M}+\mathrm{H}]^{+}$calcd. for $\mathrm{C}_{30} \mathrm{H}_{33} \mathrm{ClNO}$ : 458.2245 , found: 458.2248 .

2,3-Bis(4-(tert-butyl)phenyl)-6-methoxycarbonyl-1-methylquinolin-4(1H)-one (4f). White solid (28\%). m.p. 273.8-274.5 ${ }^{\circ} \mathrm{C} ;{ }^{1} \mathrm{H}$ NMR (500 MHz, Chloroform- $\left.d\right) \delta 9.23(\mathrm{~d}, J=2.2 \mathrm{~Hz}, 1 \mathrm{H}), 8.35(\mathrm{dd}, J=9.0,2.2 \mathrm{~Hz}$, $1 \mathrm{H}), 7.61(\mathrm{~d}, J=9.0 \mathrm{~Hz}, 1 \mathrm{H}), 7.28(\mathrm{~d}, J=2.4 \mathrm{~Hz}, 2 \mathrm{H}), 7.12-7.08(\mathrm{~m}, 2 \mathrm{H}), 7.06-7.03(\mathrm{~m}, 2 \mathrm{H}), 6.93-6.89$ $(\mathrm{m}, 2 \mathrm{H}), 3.99$ (s, 3H), $3.64(\mathrm{~s}, 3 \mathrm{H}), 1.27(\mathrm{~s}, 9 \mathrm{H}), 1.21(\mathrm{~s}, 9 \mathrm{H}) ;{ }^{13} \mathrm{C}$ NMR $(150 \mathrm{MHz}$, Chloroform-d) $\delta 176.5$, 167.0, 153.2, 152.3, 149.0, 144.4, 132.8, 132.6, 132.1, 131.1, 130.3, 129.7, 126.4, 125.9, 125.4, 125.3, 124.6, 116.4, 52.6, 38.4, 35.0, 34.6, 31.6, 31.5. HRMS (ESI) $m / z[\mathrm{M}+\mathrm{H}]^{+}$calcd. for $\mathrm{C}_{32} \mathrm{H}_{36} \mathrm{NO}_{3}$ : 482.269 , found: 482.2686 .

2,3-Bis(4-methoxyphenyl)-1-methylquinolin-4(1H)-one (4g). White solid (36\%). m.p. 192.7-193.2 ${ }^{\circ} \mathrm{C} ;{ }^{1} \mathrm{H}$ NMR (500 MHz, Chloroform- $d) \delta 8.57(\mathrm{~d}, J=8.1 \mathrm{~Hz}, 1 \mathrm{H}), 7.71(\mathrm{t}, J=8.0 \mathrm{~Hz}, 1 \mathrm{H}), 7.55(\mathrm{~d}, J=8.7 \mathrm{~Hz}$, $1 \mathrm{H}), 7.42(\mathrm{t}, J=7.5 \mathrm{~Hz}, 1 \mathrm{H}), 7.06(\mathrm{~d}, J=8.1 \mathrm{~Hz}, 2 \mathrm{H}), 6.96(\mathrm{~d}, J=8.2 \mathrm{~Hz}, 2 \mathrm{H}), 6.81(\mathrm{~d}, J=8.1 \mathrm{~Hz}, 2 \mathrm{H}), 6.68$ $(\mathrm{d}, J=8.1 \mathrm{~Hz}, 2 \mathrm{H}), 3.79(\mathrm{~s}, 3 \mathrm{H}), 3.73(\mathrm{~s}, 3 \mathrm{H}), 3.55(\mathrm{~s}, 3 \mathrm{H}) ;{ }^{13} \mathrm{C}$ NMR $(125 \mathrm{MHz}$, Chloroform-d) $\delta 176.9$, $159.9,158.1,152.4,141.9,132.8,132.5,131.3,128.7,127.9,127.8,127.0,124.5,123.8,116.2,114.2,113.5$, 55.6, 55.5, 38.1. HRMS (ESI) $m / z[\mathrm{M}+\mathrm{H}]^{+}$calcd. for $\mathrm{C}_{24} \mathrm{H}_{22} \mathrm{NO}_{3}: 372.1594$, found: 372.1596 .

6-Chloro-2,3-bis(4-methoxyphenyl)-1-methylquinolin-4(1H)-one (4h). White solid (52\%). m.p. 241.5-242.1 ${ }^{\circ} \mathrm{C} ;{ }^{1} \mathrm{H}$ NMR $(500 \mathrm{MHz}$, Chloroform-d) $\delta 8.51(\mathrm{~d}, J=2.5 \mathrm{~Hz}, 1 \mathrm{H}), 7.63(\mathrm{dd}, J=9.1,2.6 \mathrm{~Hz}, 1 \mathrm{H}), 7.50(\mathrm{~d}$, $J=9.1 \mathrm{~Hz}, 1 \mathrm{H}), 7.05(\mathrm{~d}, J=8.6 \mathrm{~Hz}, 2 \mathrm{H}), 6.94(\mathrm{~d}, J=8.6 \mathrm{~Hz}, 2 \mathrm{H}), 6.81(\mathrm{~d}, J=8.3 \mathrm{~Hz}, 2 \mathrm{H}), 6.68(\mathrm{~d}, J=8.3$ $\mathrm{Hz}, 2 \mathrm{H}), 3.79$ (s, 3H), 3.73 (s, 3H), 3.53 (s, 3H); ${ }^{13} \mathrm{C}$ NMR (125 MHz, Chloroform-d) $\delta 175.8,160.1,158.2$, 152.7, 140.3, 132.7, 132.7, 131.3, 130.0, 128.3, 128.0, 127.5, 127.0, 124.8, 118.0, 114.2, 113.6, 55.6, 55.5, 38.2. HRMS (ESI) $m / z[\mathrm{M}+\mathrm{H}]^{+}$calcd. for $\mathrm{C}_{24} \mathrm{H}_{21} \mathrm{ClNO}_{3}: 406.1204$, found: 406.1203 .

6-Methoxycarbonyl-2,3-bis(4-methoxyphenyl)-1-methylquinolin-4(1H)-one (4i). White solid (41\%). m.p. 242.3-243.7 ${ }^{\circ} \mathrm{C} ;{ }^{1} \mathrm{H}$ NMR (500 MHz, Chloroform- $\left.d\right) \delta 9.19(\mathrm{~d}, J=2.2 \mathrm{~Hz}, 1 \mathrm{H}), 8.32(\mathrm{dd}, J=9.0,2.2 \mathrm{~Hz}$, $1 \mathrm{H}), 7.58(\mathrm{~d}, J=9.0 \mathrm{~Hz}, 1 \mathrm{H}), 7.10-7.03(\mathrm{~m}, 2 \mathrm{H}), 6.98-6.92(\mathrm{~m}, 2 \mathrm{H}), 6.85-6.78(\mathrm{~m}, 2 \mathrm{H}), 6.73-6.66(\mathrm{~m}, 2 \mathrm{H})$, 3.96 (s, 3H), 3.79 (s, 3H), 3.73 (s, 3H), 3.55 (s, 3H); ${ }^{13} \mathrm{C}$ NMR (125 MHz, Chloroform-d) $\delta$ 176.8, 167.0, $160.1,158.2,152.8,144.5,132.9,132.7,131.3,130.4,128.1,127.5,126.3,125.6,125.4,116.5,114.3,113.6$, 55.6, 55.5, 52.6, 38.3. HRMS (ESI) $m / z[\mathrm{M}+\mathrm{H}]^{+}$calcd. for $\mathrm{C}_{26} \mathrm{H}_{24} \mathrm{NO}_{5}: 430.1649$, found: 430.1648 .

2,3-Bis(4-fluorophenyl)-1-methylquinolin-4(1H)-one (4j). White solid (47\%). m.p. $239.2-241.8{ }^{\circ} \mathrm{C} ;{ }^{1} \mathrm{H}$ NMR $(500 \mathrm{MHz}$, Chloroform-d) $\delta 8.56(\mathrm{dd}, J=8.1,1.7 \mathrm{~Hz}, 1 \mathrm{H}), 7.74(\mathrm{ddd}, J=8.6,6.9,1.6 \mathrm{~Hz}, 1 \mathrm{H}), 7.57$ (d, $J=8.6 \mathrm{~Hz}, 1 \mathrm{H}), 7.45(\mathrm{t}, J=7.6 \mathrm{~Hz}, 1 \mathrm{H}), 7.17-7.10(\mathrm{~m}, 2 \mathrm{H}), 7.05-6.93(\mathrm{~m}, 4 \mathrm{H}), 6.83(\mathrm{t}, J=8.8 \mathrm{~Hz}, 2 \mathrm{H})$, $3.55(\mathrm{~s}, 3 \mathrm{H}) ;{ }^{13} \mathrm{C}$ NMR $(125 \mathrm{MHz}$, Chloroform- $d) \delta 176.4,162.7(\mathrm{~d}, J=248.9 \mathrm{~Hz}), 161.5(\mathrm{~d}, J=243.6 \mathrm{~Hz})$, 151.3, 141.6, $133.1(\mathrm{~d}, J=8.0 \mathrm{~Hz}), 132.6,131.7(\mathrm{~d}, J=8.2 \mathrm{~Hz}), 131.1(\mathrm{~d}, J=3.7 \mathrm{~Hz}), 129.0(\mathrm{~d}, \mathrm{~J}=4.6 \mathrm{~Hz})$, 127.6, 126.7, 124.0, 123.7, 116.0, $115.9(\mathrm{~d}, J=13.2 \mathrm{~Hz}), 114.8(\mathrm{~d}, J=21.2 \mathrm{~Hz}), 37.8 .{ }^{19} \mathrm{~F} \mathrm{NMR}(471 \mathrm{MHz}$, Chloroform- $d$ ) $\delta-111.0,-116.2$. HRMS (ESI) $m / z[\mathrm{M}+\mathrm{H}]^{+}$calcd. for $\mathrm{C}_{22} \mathrm{H}_{16} \mathrm{~F}_{2} \mathrm{NO}$ : 348.1194, found: 348.1192 .

6-Chloro-2,3-bis(4-fluorophenyl)-1-methylquinolin-4(1H)-one (4k). White solid (68\%). m.p. 293.3-294.2 ${ }^{\circ} \mathrm{C}$; ${ }^{1} \mathrm{H}$ NMR (500 MHz, Chloroform-d) $\delta 8.51(\mathrm{~d}, J=2.6 \mathrm{~Hz}, 1 \mathrm{H}), 7.67(\mathrm{dd}, J=9.1,2.6 \mathrm{~Hz}, 1 \mathrm{H}), 7.52(\mathrm{~d}, J=$ $9.1 \mathrm{~Hz}, 1 \mathrm{H}), 7.17-7.10(\mathrm{~m}, 2 \mathrm{H}), 7.06-6.93(\mathrm{~m}, 4 \mathrm{H}), 6.84(\mathrm{t}, J=8.7 \mathrm{~Hz}, 2 \mathrm{H}), 3.54(\mathrm{~s}, 3 \mathrm{H}) ;{ }^{13} \mathrm{C} \mathrm{NMR}(125$ $\mathrm{MHz}$, Chloroform- $d$ ) $\delta 175.2,162.8(\mathrm{~d}, J=249.3 \mathrm{~Hz}), 161.5(\mathrm{~d}, J=244.1 \mathrm{~Hz}), 151.5,140.0,133.0(\mathrm{~d}, J=8.0$ $\mathrm{Hz}), 132.8,131.6(\mathrm{~d}, J=8.2 \mathrm{~Hz}), 131.3(\mathrm{~d}, J=3.5 \mathrm{~Hz}), 130.8(\mathrm{~d}, J=3.7 \mathrm{~Hz}), 130.3,126.8,124.1,117.8$, $116.0(\mathrm{~d}, J=21.7 \mathrm{~Hz}), 114.9(\mathrm{~d}, J=213 \mathrm{~Hz}), 114.8,38.0 .{ }^{19} \mathrm{~F}$ NMR $(471 \mathrm{MHz}$, Chloroform-d) $\delta-110.6$, -115.8. HRMS (ESI) $m / z[\mathrm{M}+\mathrm{H}]^{+}$calcd. for $\mathrm{C}_{22} \mathrm{H}_{15} \mathrm{ClF}_{2} \mathrm{NO}$ : 382.0805, found: 382.0795 . 
6-Methoxycarbonyl-2,3-bis(4-fluorophenyl)-1-methylquinolin-4(1H)-one (41). White solid (59\%). m.p. 213.5-214.2 ${ }^{\circ} \mathrm{C} ;{ }^{1} \mathrm{H}$ NMR $(500 \mathrm{MHz}$, Chloroform- $d$ ) $\delta 9.19(\mathrm{~d}, J=2.1 \mathrm{~Hz}, 1 \mathrm{H}), 8.35(\mathrm{dd}, J=9.0,2.2 \mathrm{~Hz}$, $1 \mathrm{H}), 7.60(\mathrm{~d}, J=9.0 \mathrm{~Hz}, 1 \mathrm{H}), 7.17-7.12(\mathrm{~m}, 2 \mathrm{H}), 7.06-6.94(\mathrm{~m}, 4 \mathrm{H}), 6.84(\mathrm{t}, J=8.7 \mathrm{~Hz}, 2 \mathrm{H}), 3.97(\mathrm{~s}, 3 \mathrm{H})$, $3.57(\mathrm{~s}, 3 \mathrm{H}) ;{ }^{13} \mathrm{C}$ NMR $(125 \mathrm{MHz}$, Chloroform- $d$ ) $\delta$ 176.5, 166.8, $163.1(\mathrm{~d}, J=249.3 \mathrm{~Hz}), 161.0(\mathrm{~d}, J=$ $244.3 \mathrm{~Hz}), 152.0,144.4,133.3(\mathrm{~d}, J=9.4 \mathrm{~Hz}), 133.1,131.9(\mathrm{~d}, J=8.3 \mathrm{~Hz}), 131.3(\mathrm{~d}, J=3.2 \mathrm{~Hz}), 130.9(\mathrm{~d}$, $J=3.6 \mathrm{~Hz}), 130.3,126.3,125.9,125.1,116.5(\mathrm{~d}, J=14.1 \mathrm{~Hz}), 116.2,115.2(\mathrm{~d}, J=21.3 \mathrm{~Hz}), 52.7,38.4 .{ }^{19} \mathrm{~F}$ NMR (471 MHz, Chloroform- $d$ ) $\delta-110.5,-115.7$. HRMS (ESI) $m / z[\mathrm{M}+\mathrm{H}]^{+}$calcd. for $\mathrm{C}_{24} \mathrm{H}_{18} \mathrm{~F}_{2} \mathrm{NO}_{3}$ : 406.1249, found: 406.1247 .

2,3-Bis(4-chlorophenyl)-1,6-dimethylquinolin-4(1H)-one (4m). White solid (68\%). m.p. $226 \cdot 1-227.3{ }^{\circ} \mathrm{C} ;{ }^{1} \mathrm{H}$ NMR (500 MHz, Chloroform- $d$ ) $\delta 8.35-8.32(\mathrm{~m}, 1 \mathrm{H}), 7.56(\mathrm{dd}, J=8.7,2.3 \mathrm{~Hz}, 1 \mathrm{H}), 7.47(\mathrm{~d}, J=8.8 \mathrm{~Hz}$, 1H), 7.32-7.28 (m, 2H), 7.14-7.07 (m, 4H), 6.99-6.92 (m, 2H), $3.52(\mathrm{~s}, 3 \mathrm{H}), 2.51(\mathrm{~s}, 3 \mathrm{H}) ;{ }^{13} \mathrm{C}$ NMR $(125$ MHz, Chloroform-d) $\delta$ 176.3, 150.9, 139.9, 135.5, 134.6, 134.4, 134.3, 133.6, 133.1, 132.6, 131.3, 129.3, 128.3, 127.1, 126.8, 123.2, 116.1, 38.0, 21.3. HRMS (ESI) $\mathrm{m} / z$ [M + H] ${ }^{+}$calcd. for $\mathrm{C}_{23} \mathrm{H}_{18} \mathrm{C}_{12} \mathrm{NO}$ : 394.0760, found: 394.0758 .

2,3-Bis(4-chlorophenyl)-6-methoxycarbonyl-1-methylquinolin-4(1H)-one (4n). White solid (74\%). m.p. 278.4-279.1 ${ }^{\circ} \mathrm{C} ;{ }^{1} \mathrm{H}$ NMR $(500 \mathrm{MHz}$, Chloroform- $d$ ) $\delta 9.17(\mathrm{~d}, J=2.1 \mathrm{~Hz}, 1 \mathrm{H}), 8.35(\mathrm{dd}, J=9.0,2.2 \mathrm{~Hz}$, $1 \mathrm{H}), 7.59(\mathrm{~d}, J=9.0 \mathrm{~Hz}, 1 \mathrm{H}), 7.36-7.29(\mathrm{~m}, 2 \mathrm{H}), 7.16-7.07(\mathrm{~m}, 4 \mathrm{H}), 6.99-6.91(\mathrm{~m}, 2 \mathrm{H}), 3.97(\mathrm{~s}, 3 \mathrm{H}), 3.55(\mathrm{~s}$, $3 \mathrm{H}) ;{ }^{13} \mathrm{C}$ NMR $(125 \mathrm{MHz}$, Chloroform- $d$ ) $\delta 176.3,166.8,151.6,144.4,135.9,133.8,133.3,133.1,133.0$, $132.9,131.2,130.3,129.5,128.4,126.3,125.9,124.6,116.5,52.7,38.4$. HRMS (ESI) $m / z[\mathrm{M}+\mathrm{H}]^{+}$calcd. for $\mathrm{C}_{24} \mathrm{H}_{18} \mathrm{C}_{12} \mathrm{NO}_{3}: 438.0658$, found: 438.0661 .

1-Methyl-2,3-diphenylquinoline-4(1H)-thione (5). Brown solid (95\%). ${ }^{1} \mathrm{H}$ NMR (400 MHz, DMSO- $\left.d_{6}\right) \delta$ $9.02(\mathrm{dd}, J=8.3,1.5 \mathrm{~Hz}, 1 \mathrm{H}), 8.00(\mathrm{~d}, J=8.7 \mathrm{~Hz}, 1 \mathrm{H}), 7.89(\mathrm{ddd}, J=8.6,7.0,1.6 \mathrm{~Hz}, 1 \mathrm{H}), 7.63-7.58(\mathrm{~m}$, 1H), 7.33-7.23 (m, 5H), 7.11-7.06 (m, 2H), 7.02-6.98 (m, 1H), 6.96-6.91 (m, 2H), 3.61 (s, 3H). ${ }^{13} \mathrm{C} \mathrm{NMR}$ $\left(125 \mathrm{MHz}, \mathrm{DMSO}-d_{6}\right) \delta 140.8,137.3,136.9,135.2,133.8,132.9,131.5,130.7,129.6,129.0,128.5,127.6$, 126.1, 126.0, 118.7, 39.2. HRMS (ESI) $m / z$ [M + H] ${ }^{+}$calcd. for $\mathrm{C}_{22} \mathrm{H}_{17} \mathrm{NS}: 327.1082$, found: 327.1072 .

4-((2-Ethoxy-2-oxoethyl)thio)-1-methyl-2,3-diphenylquinolin-1-ium bromide (6). White solid (84\%). ${ }^{1} \mathrm{H}$ NMR $\left(400 \mathrm{MHz}, \mathrm{DMSO}-d_{6}\right) \delta 8.97(\mathrm{~d}, J=8.5 \mathrm{~Hz}, 1 \mathrm{H}), 8.70(\mathrm{~d}, J=9.0 \mathrm{~Hz}, 1 \mathrm{H}), 8.38(\mathrm{t}, J=8.0 \mathrm{~Hz}, 1 \mathrm{H}), 8.20(\mathrm{t}$, $J=7.8 \mathrm{~Hz}, 1 \mathrm{H}), 7.40(\mathrm{~s}, 5 \mathrm{H}), 7.27(\mathrm{~d}, J=5.6 \mathrm{~Hz}, 3 \mathrm{H}), 7.17-7.10(\mathrm{~m}, 2 \mathrm{H}), 4.25(\mathrm{~s}, 3 \mathrm{H}), 3.95(\mathrm{q}, J=7.1 \mathrm{~Hz}$, 2H), $3.54(\mathrm{~s}, 2 \mathrm{H}), 1.05(\mathrm{t}, J=7.1 \mathrm{~Hz}, 3 \mathrm{H}) .{ }^{13} \mathrm{C}$ NMR $(125 \mathrm{MHz}$, DMSO-d 6 ) $\delta$ 168.0, 158.3, 155.2, 140.3, 138.3, 136.1, 135.7, 132.9, 131.0, 130.6, 130.5, 129.6, 129.1, 128.8, 128.6, 128.5, 121.1, 61.8, 43.7, 37.2, 14.2. HRMS (ESI) $m / z[\mathrm{M}+\mathrm{H}]^{+}$calcd. for $\mathrm{C}_{26} \mathrm{H}_{24} \mathrm{NO}_{2} \mathrm{~S}^{+}$: 414.1522, found: 414.1512 .

\section{Conclusions}

In summary, we have developed a traceless approach to quinolin-4(1H)-one derivatives through rhodium(III)-catalyzed $\mathrm{C}-\mathrm{H}$ annulation of $\mathrm{N}$-nitrosoanilines with cyclopropenones. This reaction system provides a straightforward and atom-economical route for constructing the six-membered quinolin-4(1H)-one scaffolds, which may find important synthetic applications in the construction of heterocyclic compounds in pharmaceutical chemistry.

Supplementary Materials: The following are available online: Figures S1 and S2, Control Experiments for the Mechanistic Studies; Figure S3, X-ray Crystallographic Data; Figure S4, Copies of the ${ }^{1} \mathrm{H}-\mathrm{NMR},{ }^{13} \mathrm{C}-\mathrm{NMR}$, and ${ }^{19}$ F-NMRspectra.

Author Contributions: Conceptualization, L.L.; experiments and analyses, L.L., J.L., W.D., and F.G.; writing-original draft preparation, L.L.; writing—review and editing, H.L., K.C., and Y.Z. All authors have read and agreed to the published version of the manuscript.

Funding: This research was funded by the National Natural Science Foundation of China (No.21672232, 21977106, 81620108027), National S \& T Major Projects (2018ZX09711002), and the Strategic Priority Research Program of the Chinese Academy of Sciences (XDA12040217). 
Acknowledgments: We gratefully acknowledge the financial support received from the National Natural Science Foundation of China and the Strategic Priority Research Program of the Chinese Academy of Sciences. We would like to thank all the reviewers for their great comments.

Conflicts of Interest: The authors declare no conflict of interest.

\section{References}

1. Carta, D.; Ferlin, M.G. An Overview on 2-arylquinolin-4(1H)-ones and Related Structures as Tubulin Polymerisation Inhibitors. Curr. Top. Med. Chem. 2014, 14, 2322-2345. [CrossRef] [PubMed]

2. Hadida, S.; Van Goor, F.; Zhou, J.; Arumugam, V.; McCartney, J.; Hazlewood, A.; Decker, C.; Negulescu, P.; Grootenhuis, P.D. Discovery of N-(2,4-di-tert-butyl-5-hydroxyphenyl)-4-oxo-1,4-dihydroquinoline3-carboxamide (VX-770, ivacaftor), a potent and orally bioavailable CFTR potentiator. J. Med. Chem. 2014, 57, 9776-9795. [CrossRef] [PubMed]

3. Sanchez, J.P.; Gogliotti, R.D.; Domagala, J.M.; Gracheck, S.J.; Huband, M.D.; Sesnie, J.A.; Cohen, M.A.; Shapiro, M.A. The synthesis, structure-activity, and structure-side effect relationships of a series of 8-alkoxyand 5-amino-8-alkoxyquinolone antibacterial agents. J. Med. Chem. 1995, 38, 4478-4487. [CrossRef] [PubMed]

4. Sato, M.; Motomura, T.; Aramaki, H.; Matsuda, T.; Yamashita, M.; Ito, Y.; Kawakami, H.; Matsuzaki, Y.; Watanabe, W.; Yamataka, K.; et al. Novel HIV-1 integrase inhibitors derived from quinolone antibiotics. J. Med. Chem. 2006, 49, 1506-1508. [CrossRef]

5. Wentland, M.P.; Bailey, D.M.; Cornett, J.B.; Dobson, R.A.; Powles, R.G.; Wagner, R.B. Novel amino-substituted 3-quinolinecarboxylic acid antibacterial agents: Synthesis and structure-activity relationships. J. Med. Chem. 1984, 27, 1103-1108. [CrossRef]

6. Xiao, Z.P.; Li, H.Q.; Shi, L.; Lv, P.C.; Song, Z.C.; Zhu, H.L. Synthesis, antiproliferative activity, and structure-activity relationships of 3-aryl-1H-quinolin-4-ones. ChemMedChem 2008, 3, 1077-1082. [CrossRef]

7. Soural, M.; Hradil, P.; Krupkova, S.; Hlavac, J. An Interesting Synthetic Pathway to Some Quinolin-4(1H)ones: Phenacylanthranilates Rearrangement-Limits and Scopes. Mini-Rev. Org. Chem. 2012, 9, 426-432. [CrossRef]

8. Coffman, K.C.; Palazzo, T.A.; Hartley, T.P.; Fettinger, J.C.; Tantillo, D.J.; Kurth, M.J. Heterocycle-heterocycle strategies: (2-nitrophenyl)isoxazole precursors to 4-aminoquinolines, $1 \mathrm{H}$-indoles, and quinolin-4(1H)-ones. Org. Lett. 2013, 15, 2062-2065. [CrossRef]

9. Kang, S.; Park, S.; Kim, K.S.; Song, C.; Lee, Y. Copper-Catalyzed Aza-Michael Addition of 2-Aminobenzoate to beta-Substituted $\alpha, \beta$-Unsaturated Ketones: One-Pot Synthesis of 3-Carbonyl-2-Substituted Quinolin-4(1H)-ones. J. Org. Chem. 2018, 83, 2694-2705. [CrossRef]

10. Chen, J.R.; Hu, X.Q.; Lu, L.Q.; Xiao, W.J. Exploration of Visible-Light Photocatalysis in Heterocycle Synthesis and Functionalization: Reaction Design and Beyond. Acc. Chem. Res. 2016, 49, 1911-1923. [CrossRef]

11. Zhang, B.; Studer, A. Recent advances in the synthesis of nitrogen heterocycles via radical cascade reactions using isonitriles as radical acceptors. Chem. Soc. Rev. 2015, 44, 3505-3521. [CrossRef] [PubMed]

12. Zhou, L.; Lokman Hossain, M.; Xiao, T. Synthesis of N-Containing Heterocyclic Compounds Using Visible-light Photoredox Catalysis. Chem. Rec. 2016, 16, 319-334. [CrossRef] [PubMed]

13. Akram, M.O.; Banerjee, S.; Saswade, S.S.; Bedi, V.; Patil, N.T. Oxidant-free oxidative gold catalysis: The new paradigm in cross-coupling reactions. Chem. Commun. 2018, 54, 11069-11083. [CrossRef] [PubMed]

14. Cui, X.; Mo, J.; Wang, L.; Liu, Y. Transition-Metal-Catalyzed Direct C-H Functionalization under External-Oxidant-Free Conditions. Synthesis 2015, 47, 439-459. [CrossRef]

15. Xu, P.; Li, W.; Xie, J.; Zhu, C. Exploration of C-H Transformations of Aldehyde Hydrazones: Radical Strategies and Beyond. Acc. Chem. Res. 2018, 51, 484-495. [CrossRef]

16. Li, D.D.; Cao, Y.X.; Wang, G.W. Palladium-catalyzed ortho-acyloxylation of $N$-nitrosoanilines via direct $\mathrm{sp}^{2}$ C-H bond activation. Org. Biomol. Chem. 2015, 13, 6958-6964. [CrossRef]

17. Liu, Z.; Zeng, H.; Zhang, W.; Song, C.; Yang, F.; Liu, Y.; Zhu, J. Rh(III)-catalyzed N-nitroso-directed C-H olefination polymerization. Polymer 2019, 172, 152-159. [CrossRef]

18. Peng, Q.; Hu, J.; Huo, J.; Yuan, H.; Xu, L.; Pan, X. Cp*Rh(iii) catalyzed ortho-halogenation of N-nitrosoanilines by solvent-controlled regioselective C-H functionalization. Org. Biomol. Chem. 2018, 16, 4471-4481. [CrossRef]

19. Li, L.; Wang, G.-W. Solvent-free rhodium(III)-catalyzed synthesis of 2-aminoanilides via C-H amidation of $N$-nitrosoanilines under ball-milling conditions. Tetrahedron 2018, 74, 4188-4196. [CrossRef] 
20. Liu, B.; Fan, Y.; Gao, Y.; Sun, C.; Xu, C.; Zhu, J. Rhodium(III)-catalyzed N-nitroso-directed C-H olefination of arenes. High-yield, versatile coupling under mild conditions. J. Am. Chem. Soc. 2013, 135, 468-473. [CrossRef]

21. Wu, Y.; Feng, L.J.; Lu, X.; Kwong, F.Y.; Luo, H.B. Palladium-catalyzed oxidative C-H bond acylation of $N$-nitrosoanilines with toluene derivatives: A traceless approach to synthesize $N$-alkyl-2aminobenzophenones. Chem. Commun. 2014, 50, 15352-15354. [CrossRef] [PubMed]

22. Wu, Y.; Sun, L.; Chen, Y.; Zhou, Q.; Huang, J.W.; Miao, H.; Luo, H.B. Palladium-Catalyzed Decarboxylative Acylation of $N$-Nitrosoanilines with alpha-Oxocarboxylic Acids. J. Org. Chem. 2016, 81, 1244-1250. [CrossRef] [PubMed]

23. Hu, X.; Chen, X.; Shao, Y.; Xie, H.; Deng, Y.; Ke, Z.; Jiang, H.; Zeng, W. Co(III)-Catalyzed Coupling-Cyclization of Aryl C-H Bonds with $\alpha$-Diazoketones Involving Wolff Rearrangement. ACS Catal. 2018, 8, 1308-1312. [CrossRef]

24. Liang, Y.; Jiao, N. Cationic Cobalt(III) Catalyzed Indole Synthesis: The Regioselective Intermolecular Cyclization of N-Nitrosoanilines and Alkynes. Angew. Chem. Int. Ed. 2016, 55, 4035-4039. [CrossRef]

25. Liu, B.; Song, C.; Sun, C.; Zhou, S.; Zhu, J. Rhodium(III)-catalyzed indole synthesis using N-N bond as an internal oxidant. J. Am. Chem. Soc. 2013, 135, 16625-16631. [CrossRef]

26. Yu, S.; Li, X. Rhodium(III)-catalyzed C-C coupling of arenes with 2-vinyloxiranes: Synthesis of allylic alcohols. Org. Lett. 2014, 16, 1200-1203. [CrossRef]

27. Zhao, D.; Shi, Z.; Glorius, F. Indole synthesis by rhodium(III)-catalyzed hydrazine-directed C-H activation: Redox-neutral and traceless by N-N bond cleavage. Angew. Chem. Int. Ed. 2013, 52, 12426-12429. [CrossRef]

28. Zhou, S.; Wang, J.; Zhang, F.; Song, C.; Zhu, J. A Versatile, Traceless C-H Activation-Based Approach for the Synthesis of Heterocycles. Org. Lett. 2016, 18, 2427-2430. [CrossRef]

29. Wang, J.; Wang, M.; Chen, K.; Zha, S.; Song, C.; Zhu, J. C-H Activation-Based Traceless Synthesis via Electrophilic Removal of a Directing Group. Rhodium(III)-Catalyzed Entry into Indoles from N-Nitroso and alpha-Diazo-beta-keto Compounds. Org. Lett. 2016, 18, 1178-1181. [CrossRef]

30. Song, X.; Gao, C.; Li, B.; Zhang, X.; Fan, X. Regioselective Synthesis of 2-Alkenylindoles and 2-Alkenylindole-3-carboxylates through the Cascade Reactions of $\mathrm{N}$-Nitrosoanilines with Propargyl Alcohols. J. Org. Chem. 2018, 83, 8509-8521. [CrossRef]

31. Fang, F.; Zhang, C.; Zhou, C.; Li, Y.; Zhou, Y.; Liu, H. Rh(III)-Catalyzed C-H Activation of Benzoylacetonitriles and Tandem Cyclization with Diazo Compounds to Substituted Benzo[ de]chromenes. Org. Lett. 2018, 20, 1720-1724. [CrossRef] [PubMed]

32. Li, Y.; Li, J.; Wu, X.; Zhou, Y.; Liu, H. Rh(III)-Catalyzed C-H Cyclization of Arylnitrones with Diazo Compounds: Access to 3-Carboxylate Substituted N-Hydroxyindoles. J. Org. Chem. 2017, 82, 8984-8994. [CrossRef] [PubMed]

33. Li, Y.; Zhou, J.; Fang, F.; Xu, B.; Liu, H.; Zhou, Y. Rhodium(III)-Catalyzed C-H Activation of alpha-Iminonitriles or alpha-Imino Esters and Cyclization with Acrylates to 2 H-Isoindoles. J. Org. Chem. 2018, 83, 11736-11746. [CrossRef] [PubMed]

34. Wu, X.; Wang, B.; Zhou, S.; Zhou, Y.; Liu, H. Ruthenium-Catalyzed Redox-Neutral [4 + 1] Annulation of Benzamides and Propargyl Alcohols via C-H Bond Activation. ACS Catal. 2017, 7, 2494-2499. [CrossRef]

35. Yang, Q.; Wu, C.; Zhou, J.; He, G.; Liu, H.; Zhou, Y. Highly selective C-H bond activation of $\mathrm{N}$-arylbenzimidamide and divergent couplings with diazophosphonate compounds: A catalyst-controlled selective synthetic strategy for 3-phosphorylindoles and 4-phosphorylisoquinolines. Org. Chem. Front. 2019, 6, 393-398. [CrossRef]

36. Zhou, J.; Li, J.; Li, Y.; Wu, C.; He, G.; Yang, Q.; Zhou, Y.; Liu, H. Direct Synthesis of 3-Acylindoles through Rhodium(III)-Catalyzed Annulation of N-Phenylamidines with alpha-Cl Ketones. Org. Lett. 2018, 20, 7645-7649. [CrossRef]

37. Kondo, T.; Taniguchi, R.; Kimura, Y. Ruthenium- and Rhodium-Catalyzed Ring-Opening Coupling Reactions of Cyclopropenones with Alkenes or Alkynes. Synlett 2018, 29, 717-722. [CrossRef]

38. Kong, L.; Zhou, X.; Xu, Y.; Li, X. Rhodium(III)-Catalyzed Acylation of C(sp $\left.{ }^{3}\right)-H$ Bonds with Cyclopropenones. Org. Lett. 2017, 19, 3644-3647. [CrossRef]

39. Xie, F.; Yu, S.; Qi, Z.; Li, X. Nitrone Directing Groups in Rhodium(III)-Catalyzed C-H Activation of Arenes: 1,3-Dipoles versus Traceless Directing Groups. Angew. Chem. Int. Ed. 2016, 55, 15351-15355. [CrossRef] 
40. Liu, Y.; Tian, Y.; Su, K.; Wang, P.; Guo, X.; Chen, B. Rhodium(iii)-catalyzed [3 + 3] annulation reactions of $N$-nitrosoanilines and cyclopropenones: An approach to functionalized 4-quinolones. Org. Chem. Front. 2019, 6, 3973. [CrossRef]

41. Mochida, S.; Hirano, K.; Satoh, T.; Miura, M. Synthesis of Functionalized $\alpha$-Pyrone and Butenolide Derivatives by Rhodium-Catalyzed Oxidative Coupling of Substituted Acrylic Acids with Alkynes and Alkenes. J. Org. Chem. 2009, 74, 6295-6298. [CrossRef]

42. Tamura, Y.; Bayomi, S.M.; Tsunekawa, M.; Ikeda, M. Nucleophilic Reactions of N-Unsubstituted Sulfoximines with Activated Acetylenes, Olefins, and Diphenylcyclopropenone. Chem. Pharm. Bull. 1979, 27, 2137-2142. [CrossRef]

43. Simmons, E.M.; Hartwig, J.F. On the interpretation of deuterium kinetic isotope effects in C-H bond functionalizations by transition-metal complexes. Angew. Chem. Int. Ed. 2012, 51, 3066-3072. [CrossRef] [PubMed]

44. Cai, S.; Zeng, J.; Li, Y.; Liu, X.-W. Polysubstituted pyrrole derivatives via 1,2-alkenyl migration of novel $\gamma$-amino- $\alpha, \beta$-unsaturated aldehydes and $\alpha$-diazocarbonyls. RSC Adv. 2014, 4, 7275-7278. [CrossRef]

45. Li, X.; Han, C.; Yao, H.; Lin, A. Organocatalyzed [3 + 2] Annulation of Cyclopropenones and beta-Ketoesters: An Approach to Substituted Butenolides with a Quaternary Center. Org. Lett. 2017, 19, 778-781. [CrossRef] [PubMed]

46. Vanos, C.M.; Lambert, T.H. Development of a catalytic platform for nucleophilic substitution: Cyclopropenone-catalyzed chlorodehydration of alcohols. Angew. Chem. Int. Ed. 2011, 50, 12222-12226. [CrossRef] [PubMed]

Sample Availability: Samples of the compounds are not available from the authors.

(C) 2020 by the authors. Licensee MDPI, Basel, Switzerland. This article is an open access article distributed under the terms and conditions of the Creative Commons Attribution (CC BY) license (http://creativecommons.org/licenses/by/4.0/). 